\title{
Existence Theorems for Multiple Integrals of the Calculus of Variations for Discontinuous Solutions (*).
}

\author{
L. Cesari - P. Brandi - A. Saltadori
}

\begin{abstract}
Summary. - The authors prove existence theorems for the minimum of multiple integrals of the 5alculus of variations with constraints on the derivatives in classes of BV possibly discontinuous solutions. To this effect the integrals are written in the form proposed by Serrin. Usual convexity conditions are requested, but no growth condition. Preliminary closure and semicontinuity theorems are proved which are analogous to those previously proved by Cesari in Sobolev classes. Compactness in $L_{1}$ of classes of $B V$ functions with equibounded total variations is derived from Cafiero-Fleming theorems.
\end{abstract}

\section{1. - Introduction.}

In the present paper we state and prove existence theorems of optimal solutions for multiple integrals of the calculus of variations, with constraints on the derivatives of the type

$$
\begin{gathered}
I(x)=\int_{G} F_{0}(t, x(t), D x(t)) d t \\
(t, x(t)) \in A, \quad D x(t) \in Q(t, x(t)), \quad t \in G \text { a.e. . }
\end{gathered}
$$

The solutions are sought in the class of vector valued functions of $v$ independent variables, or $x(t)=\left(x^{1}, \ldots, x^{n}\right), t=\left(t^{1}, \ldots, t^{\nu}\right) \in G$, which are of bounded variation in the sense of Cesari ([5] 1936).

These functions were used by OESARI in [5] to characterize nonparametric discontinuous surfaces whose generalized Lebesgue area is finite. The same functions were also used by CESARI in [10] (1937) for sufficient conditions for convergence almost everywhere of double and multiple Fourier series. The same functions were used by ConWAx and Smoliter [12] (1966) in the study of shock waves of weak solutions of conservation laws. These functions are also called BVO functions in the terminology of Conway and Siroller [12], Dafermos [13] and Di Perna [14]. As it

(*) Entrata in Redazione il 2 dicembre 1986.

Indirizzo degli AA.: L. CESARI: Mathematical Department, University of Michigan, Ann Arbor, Michigan, U.S.A.; P. Brandr and A. Salvadorr: Dipartimento di Matematica, Università degli Studi, Perugia (Italy). 
is well known, KRICKEBERG ([19] 1957) proved that these BVC functions (or BV functions) could equivalently be defined as those $L_{\mathbf{1}}(G)$-functions whose first order partial derivatives in the sense of distributions are finite measures. This alternative definition has been also used by a number of authors as MrRanda [20], Giusti [17], Anzellotil and Glaquinta [2]. We refer to the recent exposition (Cesari [9]) for more references on functions of bounded variations.

Our integral function is of the Serrin type [24] and it is modeled on the wellknown area functional (see CESARI [5]), that is,

$$
J(x)=\inf _{\Gamma(x)} \lim _{k \rightarrow \infty} \int_{G} F_{0}\left(t, x_{k}(t), D x_{k}(t)\right) d t=\inf _{\Gamma(x)} \lim _{k \rightarrow \infty} I\left(x_{k}\right),
$$

where the infimum is taken with respect to the class $\Gamma(x)$ of all the sequences $\left(x_{k}\right)$ of $W^{1,1}(G)$ functions converging to $x$ with respect to a suitable topology $\sigma$ and such that

$$
\left(t, x_{k}(t)\right) \in A, \quad D x_{k}(t) \in Q\left(t, x_{k}(t)\right), \quad t \in G \text { a.e. }
$$

Actually, for the sake of generality, we shall think of the $n$-vector $x=\left(x^{1}, \ldots, x^{n}\right)$ as made up of an $\alpha$-vector $y$ and an $(n-\alpha)$-vector $z$, say $x=(y, z), y=\left(x^{1}, \ldots, x^{\alpha}\right)$, $z=\left(x^{\alpha+1}, \ldots, x^{n}\right), 0 \leqslant \alpha \leqslant n$, with $y$ in $W^{1,1}(G)$ and $z$ in $\operatorname{BVC}(G), G \subset \boldsymbol{R}^{\mu}$.

Thus, in reality, we consider a mixed topology $\sigma$ for the elements $x_{k}=\left(y_{k}, z_{k}\right)$ and $x=(y, z)$ with $y, y_{k}, z_{k} \in W^{1,1}(G)$ and $z \in \mathrm{BVO}(G)$, i.e. $y_{k} \rightarrow y$ in $\left(L_{1}(G)\right)^{\alpha}$ and $D y_{k} \rightarrow D y$ weakly in $\left(L_{1}(G)\right)^{v \alpha}$, while $z_{k} \rightarrow z$ in $\left(L_{1}(G)\right)^{n-\alpha}$ and no condition is assumed on the gradient $\left(D \xi_{k_{k}}\right)$.

The method we adopt in our treatment is the direct method of the calculus of variations, based on compactness and lower semicontinuity, or lower closure (see [8]).

$\Delta s$ it is well-known, if $\partial G$ is regular, the bounded subsets of $W^{1,1}(G)$ are relatively compact in $L_{1}(G)$, and this result will be used in the present paper. Thus, in the spirit of the direct method, what we need is a suitable lower semicontinuity result.

Under convexity assumptions, in Sections 1,2 we prove elosure theorems, for BVC functions, with respect to $L_{1}(G)$ convergence and no topology on the derivatives. Such closure theorems are based on suitable extensions of the arguments used in [8] and $[11 b]$ for simple integrals and $[6,7]$ for multiple integrals. We do not discuss here quasi convexity or policonvexity.

In Section 3 we apply these closure results in order to prove the lower semicontinuity of $I$ and consequently to point out the precise comparison between $I$ and $J$. Indeed, we prove that, in general, $J(x) \geqslant I(x)$, and that $J$ is a true extension of $I$ in the sense that $J(x)=I(x)$ whenever $x \in W^{\mathbf{1}, 1}(G)$. In $[11 b]$ we showed, by an example, that $J$ can be actually larger than $I$. These conclusions are warranted by our systematic use of both alternative interpretations of the concept of BVC functions (efr. Section 2a). Note that they generalize results which are well-known for the area functional, that is, the Lebesgue area $L(x)$ of a surface $x$ is alway $\geqslant$ than 
the area integral $I(x)$, and that the equality sign holds if and only if $x$ belongs to the $W^{1,1}$ class ([24]).

Finally, in Section 4 we prove existence theorems for optimal solutions of the integral functional $J$ with constraints on the derivatives. Note that, in the particular case $\alpha=0$, all components of the solutions are BVC. We wish to point out that we require convexity conditions as expected but no growth assumptions.

Moreover, and for the sake of comparison with a previous paper of ours on simple integrals, i.e., $v=1$ ([11b]), we wish to mention that we persued there an analogous program for vector functions $x(t)=\left(x^{1}, \ldots, x^{n}\right)=(y, z), \quad t_{1} \leqslant t \leqslant t_{2}$, with $y=$ $=\left(x^{1}, \ldots, x^{\alpha}\right) \in \mathrm{AC}$ and $z=\left(x^{x+1}, \ldots, x^{n}\right) \in \mathrm{BVC}$. We also used a mixed topology, namely $y_{k} \rightarrow y$ uniformly in $\left[t_{1}, t_{2}\right]$ and $z_{k} \rightarrow z$ pointwise a.e. in $\left[t_{1}, t_{2}\right]$.

In [11b], for compactness, we used Helly's theorem which guarantees that any sequence $\left(x_{k}\right)$ of equibounded functions of a real variable with equibounded variations has a subsequence which converges everywhere in $\left[t_{1}, t_{2}\right]$. For functions of $v>1$ independent variables there is no Helly's theorem for BVC functions. However, there is a partial extension of Helly's theorem for functions of equibounded total variations in the sense of Vitali (which we shall use in Section $4 b$ ), and there is a compactness theorem in $L_{1}$ by Cafiero-Fleming for functions of equibounded total variations in the sense of BVC (which we shall use in Section $4 a$ ).

For functions of class $W^{1,1}(G)$, lower semicontinuity theorems, or lower closure theorems, have been proved, among others, by MorRex, Cesari, StaMPacchIa, Ioffe, Stoddart, Ferro, De Grorgi-Buttazzo-Dal Maso for the Lebesgue integral, and by WARNER, BRANDI-SALVADORI for the Weierstrass integral, under various conditions, as convexity and growth assumptions.

\section{2. - The closure theorems.}

a) Functions BVC and $\mathrm{ACg}$ in a bounded open set $G$ in $\boldsymbol{R}^{p}$.

We denote by $|A|$ or $|A|_{\text {v }}$ the measure of a measurable subset $A$ of $\boldsymbol{R}^{v}$. Let $G$ be an open bounded set of the $t$-space $\boldsymbol{R}^{\nu}, t=\left(t^{1}, \ldots, t^{\nu}\right)$. In the following, for the sake of brevity, we shall use the notations $t^{\prime j}=\left(t^{1}, \ldots, t^{j-1}, t^{j+1}, \ldots, t^{p}\right)=\tau$ and $\left(t^{j}, \tau\right)=\left(t^{1}, \ldots, t^{n}\right), j=1, \ldots, \nu$. Moreover, for any $j=1, \ldots, v$, let $G^{\prime j}$ denote the

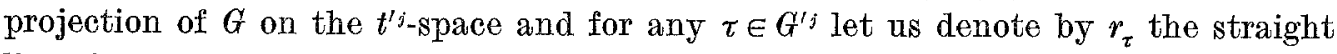
line $t^{\prime j}=\tau$. Then the intersection $G \cap r_{\tau}$ is the countable union of intervals $\left(\alpha_{s}, \beta_{s}\right)$, or $G \cap r_{\tau}=\bigcup_{s}\left(\alpha_{s}, \beta_{s}\right)$.

We say that a function $f \in L_{1}(G)$ is of bounded variation in the sense of Cesari [5] (BVC) if there exists a set $E \subset G$, with $|E|_{v}=0$, such that, for every $j=1, \ldots, v$ and for almost all $\tau \in G^{\prime j}$, the variation $V_{j s}=V\left(f,\left(\alpha_{s}, \beta_{s}\right)\right)$, computed disregarding the values taken by $f$ on $E$, are finite, $V_{j}(\tau)=\sum_{s} V_{j s}$ is finite and $V_{j} \in L_{1}\left(G^{t_{j}}\right)$. 
Then the number

$$
V^{*}(f, G)=\sum_{j=1}^{v} \int_{\alpha^{\prime} j} V_{j}(\tau) d \tau
$$

is finite and can be taken as the generalized total variation of $f$ in $G$ (cfr. [11a]).

The BVC functions which are continuous in $G$ are the classical functions of bounded variation in the sense of Tonelli (BVT) since there is no need of taking any set $E \subset G,|E|_{\nu}=0$.

If $f$ is $\mathrm{BVC}$ in $G$ and $E \subset G$ is the corresponding set of measure zero, then $E$ has intersection $E \cap l$ of linear measure zero on almost all lines $l$ parallel to the axes. Hence, $f$ is $\mathrm{BV}$ on almost all such straight lines $l$ when we disregard the values taken by $f$ on $E$, and has therefore "usual " partial derivatives $D^{j} f$ a.e. in $G$, and these derivatives are functions in $G$ of class $L_{1}(G)$. We call these $D^{j} f(t), t \in G$, $j=1, \ldots, \nu$, computed by usual incremental quotients disregarding the values taken by $f$ on $E$, the generalized first order partial derivatives on $f$ in $G$ (see [11a]), and

$$
V^{*}(j, G) \geqslant \sum_{j=1}^{v} \int_{G}\left|D^{j} f(t)\right| d t .
$$

It is well known (Serrin [23]) that if $f$ is BVC there is an equivalent function $\bar{f}$ which has partial derivatives $D^{j} \bar{f}$ a.e. in $G$ and $D^{j} \bar{f}=D^{j} f$ a.e. in $G$.

Furthermore KRICKEBERG ([19]) proved that a function $f$ is $\mathrm{BVC}$ on $G$ if and only if $f \in L_{1}(G)$ and the first order partial derivatives of $f$ in the sense of distributions are finite measures $\mu_{j}, j=1, \ldots, \nu$.

A function $f \in L_{1}(G)$ is said to be absolutely continuous in the generalized sense (AOg) if it is BVO and for every $j=1, \ldots, \nu$ and for almost all $\tau \in G^{\prime j}$, the function $f(\cdot, \tau)$ is $\mathrm{ACg}$ (i.e. it is equal a.e. to an $\mathrm{AC}$ function) on $r_{\tau}$.

The $A C g$ functions which are continuous in $G$ are the elassical functions absolutely continuous in the sense of Tonelli (ACT).

For an $\mathrm{ACg}$ function we have that

$$
V^{*}(f, G)=\sum_{j=1}^{v} \int_{G}\left|D^{j} f(t)\right| d t .
$$

Moreover it is well-known that if $f$ is $\operatorname{ACg}$ then for every $j=1, \ldots, v$ there is an equivalent function $\bar{f}_{j}$ for which the total variation $V_{j}$ is equal to the usual integral $V_{j}=\int_{j}\left|D^{j} f_{j}\right| d t$. Furthermore, KRTCKmBERG [19] showed that the ACg functions are the $f \in L_{1}(G)$ whose partial derivatives in the sense of distributions are again functions in $L_{1}(G)$. In other words, $\mathrm{ACg}$ coincides with $W^{1,1}(G)$. For more details on these concepts see [11a] and [19].

b) The properties $(Q)$ and $(F)$.

Let $n \geqslant 1, \nu \geqslant 1$ and $N \geqslant 1$ be integers. Let $A$ be a given subset of the $(t, x)$-space $\boldsymbol{R}^{\nu+n}$ and, for every $(t, x) \in A$, let $Q(t, x) \subset \boldsymbol{R}^{N}$ be given sets. Following OESARI [8], 
we shall consider the conditions on the sets $Q(t, x)$ below. We say that the sets $Q(t, x)$, $(t, x) \in A$, have property $(Q)$ at $\left(t_{0}, x_{0}\right) \in A$, with respect to $(t, x)$, provided

$$
Q\left(t_{0}, x_{0}\right)=\bigcap_{\sigma>0} \operatorname{cl} \operatorname{co} \cup\left[Q(t, x),\left|t-t_{0}\right| \leqslant \sigma,\left|x-x_{0}\right| \leqslant \sigma\right] .
$$

We say that the sets $Q(t, x),(t, x) \in A$, have property $(Q)$ at $\left(t_{0}, x_{0}\right) \in A$, with respect to $x$, provided

$$
Q\left(t_{0}, x_{0}\right)=\bigcap_{\sigma>0} \operatorname{cl} \operatorname{co} \cup\left[Q\left(t_{0}, x\right),\left|x-x_{0}\right| \leqslant \sigma\right] .
$$

We shall need below some further alternative properties of the sets $Q(t, x)$. We could say that the sets $Q(t, x),(t, x) \in A$, have property $\left(F_{1}\right)$, with respect to $x$, at the point $\left(t_{0}, x_{0}\right) \in A$, provided

$\left(F_{1}\right)$ given any number $\sigma>0$, there are constants $O=C\left(t_{0}, x_{0}, \sigma\right)>0, \delta=$ $=\delta\left(t_{0}, x_{0}, \sigma\right)>0$ such that for any $(t, x) \in A$ with $\left|t-t_{0}\right| \leqslant \sigma,\left|x-x_{0}\right|>\sigma$ and any vector $\xi \in Q(t, x)$, there are vectors $\bar{x}, \bar{\xi}$ such that

$$
(t, \vec{x}) \in A, \quad\left|\vec{x}-x_{0}\right| \leqslant \sigma, \quad \bar{\xi} \in Q(t, \bar{x}), \quad|\xi-\bar{\xi}| \leqslant C\left[|x-\bar{x}|+\left|t-t_{0}\right|\right],
$$

for $\left|t-t_{0}\right| \leqslant \delta$.

To avoid questions of measurability, however, we take the following form of this condition. We say that the sets $Q(t, x),(t, x) \in A$, have property $\left(F_{1}^{\prime}\right)$, with respect to $x$ at the point $\left(t_{0}, x_{0}\right) \in A$, provided

$\left(F_{1}^{\prime}\right)$ given any number $\sigma>0$ there are constants $C=C\left(t_{0}, x_{0}, \sigma\right)>0, \delta=$ $={ }^{F} \delta\left(t_{0}, x_{0}, \sigma\right)>0$ such that for any two measurable vector functions $x(t), \xi(t), t \in E$, on a measurable subset $E \subset G$, with $(t, x(t)) \in A,\left|x(t)-x_{0}\right|>\sigma, \xi(t) \in Q(t, x(t))$ for $\left|t-t_{0}\right| \leqslant \delta, t \in E$, there are two measurable vector functions $\bar{x}(t), \bar{\xi}(t), t \in E$, such that $(t, \bar{x}(t)) \in A,\left|\bar{x}(t)-x_{0}\right| \leqslant \sigma, \bar{\xi}(t) \in Q(t, \bar{x}(t))$ and $|\xi(t)-\bar{\xi}(t)| \leqslant C[|x(t)-\bar{x}(t)|+$ $\left.+\left|t-t_{0}\right|\right]$ for $t \in E,\left|t-t_{0}\right| \leqslant \delta$.

The conditions $\left(F_{1}\right),\left(F_{1}^{\prime}\right)$ are conditions concerning the behavior of the sets $Q(t, x)$ where $x$ is away from $x_{0}$. Note that $C$ can be as large as one wants, even for $\sigma$ small. A natural choice for $x(t)$ is of course $\bar{x}(t)=x_{0}, t \in E \cap\left\{t:\left|t-t_{0}\right| \leqslant \delta\right\}$, but the allotted generality may be useful. An analogous but essentially stronger condition is the following one, which, for the sake of brevity, we express only in terms of measurable functions.

We say that the sets $Q(t, x),(t, x) \in A$, have property $\left(F_{2}^{\prime}\right)$ at the point $\left(t_{0}, x_{0}\right) \in A$, provided

$\left(F_{2}^{\prime}\right)$ there are constants $C=C\left(t_{0}, x_{0}\right)>0, \delta=\delta\left(t_{0}, x_{0}\right)>0$ such that, for any two measurable vector functions $x(t), \xi(t), t \in E$, on a measurable subset $E \subset G$, with 
$(t, x(t)) \in A, \xi(t) \in Q(t, x(t))$ for $\left|t-t_{0}\right| \leqslant \delta, t \in E$, there is a measurable vector function $\xi(t), t \in E$, such that $\xi(t) \in Q\left(t_{0}, x_{0}\right), \quad|\xi(t)-\bar{\xi}(t)| \leqslant C\left[\left|x(t)-x_{0}\right|+\left|t-t_{0}\right|\right]$ for $t \in E,\left|t-t_{0}\right| \leqslant \delta$.

This condition $\left(F_{2}^{\prime}\right)$ actually concerns the behavior of $Q(t, x)$ for $t$ in a neighborhood of $t_{0}$ and any $x$. A variant of these conditions is the following one.

We say that the sets $Q(t, x),(t, x) \in A$, have property $\left(F_{3}^{\prime}\right)$ at the point $\left(t_{0}, x_{0}\right) \in A$, provided

$\left(F_{3}^{\prime}\right)$ given any number $\sigma>0$, there are constants $C=C\left(t_{0}, x_{0}, \sigma\right)>0, \delta=$ $=\delta\left(t_{0}, x_{0}, \sigma\right)>0$ such that for any two measurable vector functions $x(t), \xi(t), t \in E$, on a measurable subset $E \subset G$, with $(t, x(t)) \in A, \xi(t) \in Q(t, x(t)),\left|x(t)-x_{0}\right|>\sigma$ for $\left|t-t_{0}\right| \leqslant \delta, t \in E$, there are two measurable vector functions $\vec{x}(t), \vec{\xi}(t), t \in E$, such that $\left(t_{0}, \bar{x}(t)\right) \in A,\left|\vec{x}(t)-x_{0}\right| \leqslant \sigma, \quad \bar{\xi}(t) \in Q\left(t_{0}, \bar{x}(t)\right)$ and $|\xi(t)-\bar{\xi}(t)| \leqslant C\left[\left|x(t)-x_{0}\right|+\left|t-t_{0}\right|\right]$ for $t \in E,\left|t-t_{0}\right| \leqslant \delta$.

Properties $\left(F_{1}\right),\left(F_{1}^{\prime}\right),\left(F_{2}^{\prime}\right),\left(F_{3}^{\prime}\right)$ are variants of the property $(F)$ stated in 13.2.A of [8] in a different context.

Properties $(Q)$ and $\left(F_{2}^{\prime}\right)$ are trivial for $Q(t, x)=\boldsymbol{R}^{N}$ as in the elassical problems of the calculus of variations, and properties $\left(F_{1}^{\prime}\right),\left(F_{3}^{\prime}\right)$ are trivial if $Q(t, x)=\boldsymbol{R}^{N}$ and $A=G \times A_{0}, A_{0} \subset \boldsymbol{R}^{n}$ a fixed set.

\section{c) Statement of the closure theorems.}

Let $G \subset \boldsymbol{R}^{v}$ be an open bounded set and let $A \subset \boldsymbol{R}^{\nu+n}$ be such that the projection of $A$ on the $t$-space $\boldsymbol{R}^{v}$ contains $G$. We shall denote by $x(t)=\left(x^{1}(t), \ldots, x^{n}(t)\right), t \in G$, a vector function on $G$, whose components $x^{i}$ are BVC on $G$. Therefore, the usual partial derivatives $D^{i} x^{i}, j=1, \ldots, v, i=1, \ldots, n$, exist a.e. in $G$ and are $L$-integrable functions in $G$. For every $i=1, \ldots, n$, we shall denote by $\{j\}_{i}$ a given finite system $\left(j_{1}, \ldots, j_{s}\right)$ of indices $j, 1 \leqslant j_{1}<\ldots<j_{s} \leqslant \nu$, and we shall denote by $D x$ the vector of all the first order derivatives $D^{j} x^{i}, j \in\{j\}_{i}, i=1, \ldots, n$. Let $N$ denote the total number of indices contained in the $n$ systems $\{j\}_{i}, i=1, \ldots, n$. For every $(t, x) \in A$, let $Q(t, x)$ be a subset of $\boldsymbol{R}^{N}$. We shall consider the orientor field equation

$$
(t, x(t)) \in A, \quad D x(t) \in Q(t, x(t)), \quad \text { a.e. in } G,
$$

that is, the problem of determining an $n$-vector function $x(t)=\left(x^{1}, \ldots, x^{n}\right), t \in G$, whose components $x^{i}$ are BVC on $G$ and satisfies (*) a.e. in $G$.

Theorem 1 (A closure theorem). - Let assume that (i) $A$ is closed; (ii) the sets $Q(t, x),(t, x) \in A$, are closed and convex; (iii) the sets $Q(t, x),(t, x) \in A$, have property $(Q)$ with respect to $(t, x)$, and property $\left(H_{1}^{\prime}\right)$ with respect to $x$ at every point $\left(t_{0}, x_{0}\right) \in A$, with the exception perhaps of a set of points whose $t$-coordinates lie in a set $H$ of 
measure zero in the $t$-space. Let $x_{k}(t)$, or $x_{k}: G \rightarrow \boldsymbol{R}^{n}$, be a sequence of ACg solutions of the orientor field (iv) $\left(t, x_{k}(t)\right) \in A, D x_{k}(t) \in Q\left(t, x_{k}(t)\right)$ a.e. in $G, k \in N$; and assume that $(\mathrm{V}) x_{k} \rightarrow x$ in $\left(L_{1}(G)\right)^{n}$ and $x$ is BVC. Then the vector function $x$ is a solution of the orientor field equation

$$
(t, \infty(t)) \in A, \quad D x(t) \in Q(t, \infty(t)) \quad \text { a.e. in } G
$$

THEOREM 2 (A closure theorem). - The same as in Theorem 1 where (iii) is replaced by (iii)' the sets $Q(t, x),(t, x) \in A$, have property $\left(F_{2}^{\prime}\right)$ at every point $\left(t_{0}, x_{0}\right) \in A$, with the exception perhaps of a set of points whose t-coordinates lie in a set $H$ of measure zero in the $t$-space.

THEOREM 3 (A closure theorem). - The same as in Theorem 1 where (iii) is replaced by (iii)" the sets $Q(t, x),(t, x) \in A$ have property $(Q)$ with respect to $x$ only and property $\left(F_{3}^{\prime}\right)$ at every point $\left(t_{0}, x_{0}\right) \in A$ with the exception perhaps of a set of points whose $t$-coordinates lie in a set $H$ of measure zero in the $t$-space.

d) Proofs of the closure theorems.

Proof of Theorem 1. - By the hypothesis (v) we have that

$$
\lim _{k \rightarrow \infty} \int_{G}\left|x_{k}(t)-x(t)\right| d t=0
$$

If we denote by $G^{j}$ the projection of $G$ on the $t^{j}$-axis, and for every $\bar{t}^{j} \in G^{j}, \bar{t}^{j}$ scalar, we consider the intersection set $G\left(\bar{t}^{j}\right)=\left\{\tau \in \boldsymbol{R}^{\nu-1} \mid\left(\bar{t}^{j}, \tau\right) \in G\right\}$, then we know that, for a.a. $\vec{t}^{j} \in G^{j}$ we also have

$$
\lim _{k \rightarrow \infty} \int_{G\left(\bar{t} \bar{t}^{j}\right)}\left|x_{k}\left(\tilde{t}^{j}, \tau\right)-x\left(\bar{t}^{j}, \tau\right)\right| d \tau=0, \quad j=1, \ldots, \nu,
$$

where $d \tau=d t^{1} \ldots d t^{j-1} d t^{j+1} \ldots d t^{v}$. That is, for every $j=1, \ldots, v$, there is a set $E^{j} \subset G^{j}$ with $\left|E^{j}\right|_{1}=0$ such that, for all $\bar{t}^{j} \in G^{j}-E^{j}$, relation (2) holds.

Let $E_{1}^{j}=\left\{t=\left(\bar{t}^{j}, \tau\right): \bar{t}^{j} \in E^{j}, \tau \in \boldsymbol{R}^{v-1}\right\} \subset \boldsymbol{R}^{\nu}$, so that $\left|E_{1}^{j}\right|_{v}=0, j=1, \ldots, v$, and we take $E_{1}=\bigcup_{j=1}^{v} E_{1}^{j}$. Then $\left|E_{1}\right|_{v}=0$.

By hypothesis, $x$ is BVC. Let us denote by $\mu_{i j}$ the $\nu n$ finite measures which represent the first order partial derivatives of $x$ in the sense of distributions. For every $t_{0} \in \boldsymbol{R}^{v}$ let $q=\left[t_{0}-h, t_{0}+h\right]$ denote the cube $q=\left[t_{0}^{j}-h \leqslant t^{j} \leqslant t_{0}^{j}+h, j=1, \ldots, v\right]$, $h>0$.

Then by [19] and [23] (pg. 119 and 366) for almost all $h>0$ we have

$$
\int_{\alpha^{\prime j}}\left[x^{i}\left(t_{0}^{j}-h, \tau\right)-x^{i}\left(t_{0}^{j}+h, \tau\right)\right] d \tau=\mu_{i j}\left(t_{0}-h, t_{0}+h\right],
$$


where $q^{\prime s}=\left[t_{0}^{s}-h \leqslant t^{s} \leqslant t_{0}^{s}+h, s=1, \ldots, v, s \neq j\right] \subset \boldsymbol{R}^{v-1}$, and

$$
\mu_{i j}\left(t_{0}-h, t_{0}+h\right]=\int_{\alpha} D^{j} x^{i}(t) d t+S_{i j}\left(t_{0}-h, t_{0}+h\right],
$$

where $\mathbb{S}_{i j}$ is the singular part of the measure $\mu_{i j}, i=1, \ldots, n, j=1, \ldots, \nu$. As a consequence, for almost all $t_{0} \in G$ we have

$$
\lim _{h \rightarrow 0^{+}}(2 \bar{h})^{-\nu} S_{i j}\left(t_{0}-h, t_{0}+h\right]=0
$$

Let us denote by $E_{2}$, with $\left|E_{2}\right|_{p}=0$, the set of all $t_{0} \in \boldsymbol{R}^{v}$ where (5) does not hold.

Now the functions $D^{j} x^{i}$ are $L$-integrable in $G$, hence

$$
\lim _{h \rightarrow 0^{+}}(2 h)^{-p} \int_{a} D^{j} x^{i}(t) d t=D^{j} x^{i}\left(t_{0}\right) \text { a.e. in } G, \quad j=1, \ldots, v, i=1, \ldots, n
$$

Let $E_{3}$ be a set, with $\left|E_{3}\right|_{\nu}=0$, such that $(6)$ is true for all $t_{0} \in G-E_{3}$. Finally $x \in\left(L_{1}(G)\right)^{n}$, hence there is a set $E_{4} \subset G$ with $\left|E_{4}\right|_{p}=0$ such that, for all $t_{0} \in G-E_{4}$ we have

$$
\lim _{h \rightarrow 0^{+}}(2 h)^{-\nu} \int_{q}\left|x^{i}(t)-x^{i}\left(t_{0}\right)\right| d t=0, \quad i=1, \ldots, n
$$

Now by hypothesis ( $v)$ we deduce that there is a subsequence, say still $(k)$ for the sake of semplicity, such that $x_{k} \rightarrow x$ pointwise a.e. in $G$. Then by Egoroff's and Lusin's theorems, given $\lambda>0$ there is a compact set $K \subset \theta$, with $|G-K|_{v}<\lambda$ such that $x$ restricted to $K$ is continuous, hence uniformly continuous, and $x_{k} \rightarrow x$ uniformly on $K$. Thus, given $0<\sigma \leqslant 1$, there is a number $0<\delta_{1}=\delta_{1}(\sigma) \leqslant \sigma$ such that

$$
\left|x\left(t^{\prime}\right)-x\left(t^{\prime \prime}\right)\right|<\sigma / 2 \quad \text { for all } t^{\prime}, t^{\prime \prime} \in K, \quad\left|t^{\prime}-t^{\prime \prime}\right|<\delta_{1},
$$

and an integer $k_{1}=k_{1}(\sigma)$ such that

$$
\left|x_{k}(t)-x(t)\right|<\sigma / 2 \quad \text { for all } t \in K \text { and } k \geqslant k_{1} .
$$

Note that almost all points of $K$ are points of density one for $K$. As a consequence, for almost all $t_{0} \in K$ we have

$$
\lim _{h \rightarrow 0^{+}}(2 h)^{-y}|q \cap K|_{\nu}=1 .
$$

Let $E_{5}$ denote the set of all points $t_{0} \in K$ which do not have this property, hence $\left|E_{5}\right|_{v}=0$. 
For $E=E_{1} \cup E_{2} \cup E_{3} \cup E_{4} \cup E_{5} \cup H$, then $|E|_{p}=0$.

Let $t_{0}$ be any point in $K-E$ and let $\delta_{0}$ denote its distance from $\partial G$. Then conditions (2), (5), (6) and (7) hold.

Take $x_{0}=x\left(t_{0}\right)=\left(x_{0}^{1}, \ldots, x_{0}^{n}\right)$ and let $q=\left[t_{0}-h, t_{0}+h\right]$ denote any cube with $0<h<\min \left(\delta_{0}, \delta_{1}\right) / \sqrt{ } \bar{y}$, then $q \subset G$ and

$$
\left|x(t)-x_{0}\right|<\sigma / 2 \quad \text { for all } t \in q \cap K .
$$

Let $0<\delta \leqslant 1$ and $\sigma>0$ be the constants corresponding to $\left(t_{0}, x_{0}\right)$ and $\sigma$, given by the hypothesis $\left(F_{1}^{\prime}\right)$. Given $\varrho>0$, there is $0<h_{0}=h_{0}\left(t_{0}, \varrho, O\right) \leqslant \min \left(\delta_{0}, \delta_{1}, \delta\right) / 2 \sqrt{ } \bar{v}$ such that for almost all $0<h<h_{0}$ the following relations hold for $j=1, \ldots, v$, $i=1, \ldots, n$ (see $(10),(6),(5)$ and $(7))$

$$
\begin{aligned}
& (2 h)^{-v}|q-K|_{p}<\varrho / 14 C, \\
& \left|(2 h)^{-v} \int_{a} D^{j} x^{i}(t) d t-D^{i} x^{i}\left(t_{0}\right)\right| d t<\varrho / 7 n N, \\
& (2 h)^{-p}\left|S_{i j}\left(t_{0}-h, t_{0}+h\right]\right|<\varrho / 7 n N \\
& (2 h)^{-v} \int_{q}\left|x(t)-x_{0}\right| d t<\varrho / 7 C .
\end{aligned}
$$

Note that $t_{0}^{j} \notin E^{j}, j=1, \ldots, v$, and we can choose $0<h<h_{0}$ so that relation (3) holds and also $t_{0}^{j} \pm \hbar \notin E^{j}$. Having fixed $h$ relation (2) holds for $t_{0}^{j}-\hbar$ and $t_{0}^{j}+h$, hence we can take an integer $k_{0}=k_{0}\left(\varrho, h, t_{0}\right)$ sufficiently large so that

$$
(2 h)^{-\nu} \int_{\alpha^{\prime j}}\left|x_{k}^{i}\left(t_{0}^{j} \pm h, \tau\right)-x^{i}\left(t_{0}^{j} \pm h, \tau\right)\right| d \tau<\varrho / 7 n N
$$

for all $j=1, \ldots, v, i=1, \ldots, n$ and $k>k_{0}$. Finally, because of (1), we can take $\bar{k}=\bar{k}(C, \varrho, h)>\max \left(k_{1}, k_{0}\right)$ so that for $k>\bar{k}$ we also have

$$
(2 h)^{-p} \int_{Q}\left|x_{k}(t)-x(t)\right| d t<\varrho / 70 .
$$

On the other hand, by (3), (4), (15) and (13), we have

$$
\begin{gathered}
\left|(2 h)^{-\nu} \int_{a} D^{j} x^{i}(t) d t-(2 h)^{-\nu} \int_{a} D^{i} x_{k}^{i}(t) d t\right|= \\
=\left|(2 h)^{-v} \int_{\alpha^{\prime j}}\left[x^{i}\left(t_{0}^{j}+h, \tau\right)-x^{i}\left(t_{0}^{j}-h, \tau\right)-x_{k}^{i}\left(t_{0}^{j}+h, \tau\right)+x_{k}^{i}\left(t_{0}^{j}-h, \tau\right)\right] d \tau\right|- \\
-(2 h)^{-\nu}\left|S_{i j}\left(t_{0}-h, t_{0}+h\right]\right| \leqslant(2 h)^{-v} \int_{\alpha^{\prime j}}\left|x_{k}^{i}\left(t_{0}^{j}+h, \tau\right)-x^{i}\left(t_{0}^{j}+h, \tau\right)\right| d \tau+ \\
+(2 h)^{-v} \int_{q^{\prime} j}\left|x_{k}^{i}\left(t_{0}^{j}-h, \tau\right)-x^{i}\left(t_{0}^{j}-h, \tau\right)\right| d \tau+(2 h)^{-v}\left|S_{i j}\left(t_{0}-h, t_{0}+h\right]\right| \leqslant \\
\quad \leqslant \varrho / 7 n N+\varrho / 7 n N+\varrho / 7 n N=3 \varrho / 7 n N,
\end{gathered}
$$


and from (12) we deduce that, for every $k>\bar{k}$

$$
\left|D x\left(t_{0}\right)-(2 h)^{-v} \int_{a} D x_{k}(t) d t\right| \leqslant N[3 \varrho / 7 N+\varrho / 7 N]=4 \varrho / 7 .
$$

By hypothesis (iv) we know that

$$
D x_{k}(t) \in Q\left(t, x_{k}(t)\right) \quad \text { a.e. in } G \text {. }
$$

When $t$ describes the interval $q=\left[t_{0}-h ; t_{0}+h\right]$, certainly $\left|t-t_{0}\right|<2 h \sqrt{v}$, with $0<h<h_{0} \leqslant \min \left(\delta_{0}, \delta_{1}, \delta\right) / 2 \sqrt{v}$.

Hence, for all $t \in q \cap K$ and $t>\vec{k}$ we have (see (8) and (9)) $\left|t-t_{0}\right|<\delta_{1}<\sigma$, $\left|x(t)-x_{0}\right|<\sigma / 2$ and $\left|x_{k}(t)-x(t)\right|<\sigma / 2$, thus $\left|x_{k}(t)-x_{0}\right|<\sigma$.

Now we enlarge the set $q \cap K$ into the maximal subset $\tilde{K}_{k}$ of $q$ where

$$
\left|x_{k}(t)-x_{0}\right| \leqslant \sigma
$$

Certainly $\widetilde{K}_{k}$ is measurable, and then for $t \in q-\tilde{K}_{k},\left|t-t_{0}\right|<\sigma$, we have

$$
\left|x_{k}(t)-x_{0}\right|>\sigma
$$

Moreover, for $\xi_{k}(t)=D x_{k}(t)$, we certainly have

$$
\xi_{k}(t)=D x_{k}(t) \in Q\left(t, x_{k}(t)\right), \quad t \in q \text { a.e. }
$$

By virtue of property $\left(F_{1}^{i}\right)$, corresponding to $\left(t_{0}, x_{0}\right), \sigma>0$ and the functions $x_{k}(t)$, $\xi_{k}(t), t \in q-\tilde{K}_{k}$, we deduce that there are measurable functions $\bar{x}_{k}(t), \bar{\xi}_{k}(t), t \in q-\tilde{K}_{k}$, such that

$$
\begin{aligned}
\left(t, \vec{x}_{k}(t)\right) \in A, \quad\left|\bar{x}_{k}(t)-x_{0}\right| \leqslant \sigma, \quad \bar{\xi}_{k}(t) \in Q\left(t, \vec{x}_{k}(t)\right) \text { and } \\
\left|\xi_{k}(t)-\vec{\xi}_{k}(t)\right| \leqslant O\left[\left|x_{k}(t)-\bar{x}_{k t}(t)\right|+\left|t-t_{0}\right|\right], \quad t \in q-\tilde{K}_{k} .
\end{aligned}
$$

Moreover we can extend $\bar{x}_{k}$ and $\bar{\xi}_{k}$ to all $q$, by putting

$$
\bar{\xi}_{k k}(t)=\xi_{k}(t) \in Q\left(t, x_{k}(t)\right), \quad \bar{x}_{k}(t)=x_{k}(t), \quad t \in \tilde{K}_{k} .
$$

Thus by $(20),(21),(16),(14)$ and (11) we have, for $k>\bar{k}$,

$$
\begin{aligned}
& \left|(2 h)^{-v} \int_{\alpha} \xi_{k_{k}}(t) d t-(2 h)^{-\nu} \int_{Q} \xi_{k}(t) d t\right| \leqslant(2 h)^{-\nu} \int_{\alpha-\overline{\mathbb{K}}_{k}}\left|\tilde{\xi}_{k}(t)-\xi_{k}(t)\right| d t \leqslant \\
& \leqslant C(2 h)^{-\nu} \int_{\alpha-\tilde{\mathbb{K}}_{k}}\left[\left|x_{k}(t)-\bar{x}_{k l}(t)\right|+\left|t-t_{0}\right|\right] d t \leqslant
\end{aligned}
$$




$$
\begin{aligned}
& \leqslant C(2 h)^{-\nu} \int_{Q-\tilde{K}_{k}}\left[\left|x_{k}(t)-x(t)\right|+\left|x(t)-x_{0}\right|+\left|\vec{x}_{k}(t)-x_{0}\right|+\left|t-t_{0}\right|\right] d t \leqslant \\
& \left.\leqslant C(2 h)^{-\nu}\left[\int_{a} \mid x_{k}(t)-x(t)\right] d t+\int_{Q}\left|x(t)-x_{0}\right| d t+\int_{q-\tilde{K}_{k}}\left|\vec{x}_{k}(t)-x_{0}\right| d t+\int_{q-K}\left|t-t_{0}\right| d t\right] \leqslant \\
& \leqslant C[\varrho / 7 C+\varrho / 7 C+\varrho / 7 C]=3 \varrho / 7 .
\end{aligned}
$$

From (19), (18) and (22) for $k>\bar{k}$ we have

$$
\begin{aligned}
& \left|D x\left(t_{0}\right)-(2 h)^{-\nu} \int_{a} \bar{\xi}_{k}(t) d t\right| \leqslant \\
& \leqslant\left|D x\left(t_{0}\right)-(2 h)^{-\nu} \int_{a} \xi_{k}(t) d t\right|+\left|(2 h)^{-p} \int_{q} \xi_{k}(t) d t-(2 h)^{-\nu} \int_{q} \bar{\xi}_{k}(t) d t\right| \leqslant 4 \varrho / 7+3 \varrho / 7=\varrho,
\end{aligned}
$$

with (see (9), $(8)^{\prime},(20)$ and $\left.(21)\right)$

$$
\begin{gathered}
\bar{\xi}_{k}(t)=\xi_{k}(t) \in Q\left(t, x_{k}(t)\right), \quad\left|t-t_{0}\right|<\sigma, \quad\left|x_{k}(t)-x_{0}\right| \leqslant \sigma, \quad t \in \widetilde{K}_{k}, \\
\bar{\xi}_{k}(t) \in Q\left(t, \bar{x}_{k}(t)\right), \quad\left|t-t_{0}\right|<\sigma, \quad\left|\vec{x}_{k}(t)-x_{0}\right| \leqslant \sigma, \quad t \in q-\tilde{K}_{k} .
\end{gathered}
$$

Hence

$$
(2 \hbar)^{-v} \int_{q} \bar{\xi}_{k}(t) d t \in \operatorname{clco} \cup\left[Q\left(t^{\prime}, x^{\prime}\right)\left|t^{\prime}-t_{0}\right| \leqslant \sigma,\left|x^{\prime}-x_{0}\right| \leqslant \sigma\right]=\operatorname{clco} Q\left(t_{0}, x_{0}, \sigma\right),
$$

and (23) yields

$$
D x\left(t_{0}\right) \in\left[\operatorname{cl} \operatorname{co} Q\left(t_{0}, x_{0}, \sigma\right)\right]_{e} .
$$

By the arbitrariety of $\varrho>0$, from (24) we deduce that

$$
D x\left(t_{0}\right) \in \operatorname{clco} Q\left(t_{0}, x_{0}, \sigma\right)
$$

which gives, by virtue of hypothesis $(Q)$

$$
D x\left(t_{0}\right) \in \bigcap_{\sigma>0} \operatorname{clco} Q\left(t_{0}, x_{0}, \sigma\right)=Q\left(t_{0}, x_{0}\right)
$$

Thus we have $D x\left(t_{0}\right) \in Q\left(t_{0}, x\left(t_{0}\right)\right)$ a.e. in $K$ with $|G-K|_{\nu}<\lambda$, Finally, by the arbitrariety of $\lambda>0$, we have

$$
D x(t) \in Q(t, x(t)) \quad \text { a.e. in } G \text {. }
$$

Theorem 1 is thereby proved. 
Proof of Theorin 2. - The proof is the same as for Theorem 1 , though here the numbers $\delta>0$ and $C>0$ depend only on $t_{0}$ and $x_{0}=x\left(t_{0}\right)$.

Moreover we choose $h>0$ in such a way it satisfies all requirements listed in the proof of Theorem 1 , and in addition $2 \nu C h<\varrho / 7$. We recall that for every $k>\bar{k}$, we have

$$
\begin{aligned}
& (2 h)^{-v} \int_{q}\left|x(t)-x_{0}\right| d t<\varrho / 7 C \\
& (2 h)^{-v} \int_{q}\left|x_{k}(t)-x(t)\right| d t<\varrho / 7 C \\
& \left|D x\left(t_{0}\right)-(2 h)^{-\nu} \int_{Q} D x_{k}(t) d t\right|<4 \varrho / 7
\end{aligned}
$$

Moreover, by the hypotheses,

$$
\left(t, x_{k}(t)\right) \in A, \quad \xi_{l}(t)=D x_{l_{k}}(t) \in Q\left(t, x_{k}(t)\right) \quad \text { a.e. in } q
$$

By property $\left(F_{2}^{\prime}\right)$ corresponding to $\left(t_{0}, x_{0}\right)$ and the functions $x_{k}(t), \xi_{k}(t), t \in q$, there is a measurable vector function $\bar{\xi}_{k}(t), t \in q$, such that

$$
\xi_{k}(t) \in Q\left(t_{0}, x_{0}\right), \quad\left|\xi_{k}(t)-\bar{\xi}_{k}(t)\right| \leqslant O\left[\left|x_{z_{i}}(t)-x_{0}\right|+\left|t-t_{0}\right|\right], \quad t \in q .
$$

From $(20)^{\prime},(14)$ and $(16)$ we deduce that, for every $k>\bar{k}$,

$$
\begin{aligned}
& \left|(2 h)^{-v} \int_{q} \xi_{k}(t) d t-(2 h)^{-v} \int_{Q} \xi_{k}(t) d t\right| \leqslant C(2 h)^{-v} \int_{Q}\left[\left|x_{l}(t)-x_{0}\right|+\left|t-t_{0}\right|\right] d t \leqslant \\
& \leqslant C 2 \varrho / 7 C+O(2 h)^{-v}(2 h)^{v} 2 h v=2 \varrho / 7+2 h v C<3 \varrho / 7 \text {. }
\end{aligned}
$$

From (18) and $(22)^{\prime}$ we have now, for $k>\bar{k}$,

$$
\left|D x\left(t_{0}\right)-(2 h)^{-y} \int_{Q} \vec{\xi}_{k}(t) d t\right| \leqslant \varrho
$$

with $\vec{\xi}_{r}(t) \in Q\left(t_{0}, x_{0}\right)$ a.e. in $q$. Hence,

$$
(2 h)^{-\nu} \int_{Q} \xi_{k}(t) d t \in \operatorname{clco} Q\left(t_{0}, x_{0}\right)=Q\left(t_{0}, x_{0}\right)
$$

and $(23)^{\prime}$ yields

$$
D x\left(t_{0}\right) \in\left[Q\left(t_{0}, x_{0}\right)\right]_{\varrho}
$$

Since $\varrho$ is arbitrary and $Q\left(t_{0}, x_{0}\right)$ is closed, we have

$$
D x\left(t_{0}\right) \in Q\left(t_{0}, x_{0}\right)=Q\left(t_{0}, x\left(t_{0}\right)\right)
$$


This relation holds for a.e. $t_{0} \in K$, with $|G-K|_{p}<\lambda$; since $\lambda>0$ is arbitrary, we have $D x(t) \in Q(t, x(t))$ a.e. in $G$ and the proof is complete.

Proof of Theorem 3. - Is analogous to the two previous ones.

\section{3. - An application of the closure theorems.}

(a) The orientor field equation.

Let $R_{0}=\left[a_{0}, b_{0}\right]=\left[a_{0}^{j} \leqslant t^{j} \leqslant b_{0}^{j}, j=1, . ., \nu\right]$ be a closed interval such that $G \subset R_{0}$, and let $x^{0}(t), t \in R_{0}$ or $x^{0}: R_{0} \rightarrow \boldsymbol{R}$ be a given scalar function. For any interval $R=[a, b] \subset R_{0}$, we consider the usual differences of order $\nu$ relative to the $2^{\nu}$ vertices of $R$, say

$$
\begin{aligned}
& \Delta_{R} x^{0}=x^{0}(b)-x^{0}(a) \quad \text { if } \nu=1, \\
& \Delta_{R} x^{0}=x^{0}\left(b^{1}, b^{2}\right)-x^{0}\left(b^{1}, a^{2}\right)-x^{0}\left(a^{1}, b^{2}\right)+x^{0}\left(a^{1}, a^{2}\right) \quad \text { if } \nu=2,
\end{aligned}
$$

and so on.

As is well known, the function $x_{0}$ is said to be of bounded variation in the sense of Vitali (VBV) [22] if the interval function $\Delta_{R} x^{0}$ is BV, i.e.,

$$
\sup _{D} \sum_{R \in D}\left|\Delta_{R} x^{0}\right|<+\infty
$$

where the sup is taken over all the finite partitions $D$ of $R_{0}$ in nonoverlapping intervals. A VBV function has almost everywhere superficial derivative, say

$$
D^{*} x^{0}\left(t_{0}\right)=\lim _{h \rightarrow 0}(2 h)^{-r} \Delta_{q} x^{0}, \quad t_{0} \in R_{0},
$$

where $q=\left[t_{0}-h, t_{0}+h\right]$, and $D^{*} x^{0}(t)$ is an $L$-integrable function in $R_{0}$.

The function $x_{0}$ is said to be absolutely continuous in the sense of Vitali (VAC) [22] if the interval function $\Delta_{R} x^{0}$ is AC. In this case we have $\Delta_{R} x^{0}=\int_{R} D^{*} x^{0}(t) d t$.

Now we denote by $\tilde{x}(t)=\left(x^{0}, x\right)=\left(x^{0}, x^{1}, \ldots, x^{n}\right), t \in G$, a vector function of $t \in G$, where $x^{0}$ is VBV and $x^{i}, i=1, \ldots, n$, is BVC in $G$.

For every $(t, x) \in A \subset \boldsymbol{R}^{n+1}$ let $\widetilde{Q}(t, x) \subset \boldsymbol{R}^{x+1}$ be a given set. We shall consider the following field equation

$$
(t, x(t)) \in A, \quad\left(D^{*} x^{0}(t), D x(t)\right) \in \widetilde{Q}(t, x(t)) \quad \text { a.e. in } G .
$$

(b) The properties $\left(\tilde{F}^{\prime}\right)$.

Property $(Q)$ for the set $\widetilde{Q}(t, x)$ does not need a new definition. Instead, we shall formulate conditions $\left(\widetilde{F}_{1}^{\prime}\right),\left(\widetilde{F}_{2}^{\prime}\right)$ and $\left(\widetilde{F}_{3}^{\prime}\right)$ for the sets $\widetilde{Q}(t, x)$, analogous to the con- 
ditions $\left(F_{1}^{\prime}\right),\left(F_{2}^{\prime}\right)$ and $\left(F_{3}^{\prime}\right)$ of Section $2 b$. Again, to avoid questions of measurability, we express these conditions in terms of vector functions.

We say that the sets $\widetilde{Q}(t, x),(t, x) \in A$, have property $\left(\tilde{F}_{1}^{\prime}\right)$, with respect to $x$ at the point $\left(t_{0}, x_{0}\right) \in A$, provided

$\left(\tilde{F}_{1}^{\prime}\right)$ given any number $\sigma>0$, there are constants $C=C\left(t_{0}, x_{0}, \sigma\right)>0, \delta=$ $=\delta\left(t_{0}, x_{0}, \sigma\right)>0$ such that for any set of measurable vector functions $x(t), \eta(t)$, $\xi(t), t \in E$, on a measurable subset $E \subset G$ with $(t, x(t)) \in A,\left|x(t)-x_{0}\right|>\sigma$, $(\eta(t), \xi(t)) \in \widetilde{Q}(t, x(t))$ for $t \in E,\left|t-t_{0}\right| \leqslant \sigma$, there are measurable functions $\bar{x}(t), \tilde{\eta}(t)$, $\bar{\xi}(t), t \in E$, such that

$$
\begin{gathered}
(t, \vec{x}(t)) \in A, \quad\left|\vec{x}(t)-x_{0}\right| \leqslant \sigma, \quad(\bar{\eta}(t), \bar{\xi}(t)) \in \widetilde{Q}(t, \vec{x}(t)), \\
|\xi(t)-\bar{\xi}(t)| \leqslant C\left[|x(t)-\bar{x}(t)|+\left|t-t_{0}\right|\right], \\
\bar{\eta}(t) \leqslant \eta(t)+C\left[|x(t)-\bar{x}(t)|+\left|t-t_{0}\right|\right] \quad \text { for } t \in E, \quad\left|t-t_{0}\right| \leqslant \delta .
\end{gathered}
$$

The most natural choice for $\bar{x}$ is $\bar{x}(t)=x_{0}, t \in E \cap\left\{t:\left|t-t_{0}\right| \leqslant \delta\right\}$.

We say that the sets $\widetilde{Q}(t, x),(t, x) \in A$, have property $\left(\widetilde{F}_{2}^{\prime}\right)$ at the point $\left(t_{0}, x_{0}\right) \in A$, provided

$\left(\tilde{F}_{2}^{\prime}\right)$ there are constants $C=C\left(t_{0}, x_{0}\right)>0$ and $\delta=\delta\left(t_{0}, x_{0}\right)>0$ such that for any set of measurable vector functions $x(t), \eta(t), \xi(t), t \in E$, on a measurable subset $E \subset G$, with $(t, x(t)) \in A,(\eta(t), \xi(t)) \in \widetilde{Q}(t, x(t))$ for $\left|t-t_{0}\right|<\delta, t \in E$, there are two measurable vector functions $\bar{\eta}(t), \bar{\xi}(t), t \in E$, such that

$$
\begin{gathered}
(\tilde{\eta}(t), \tilde{\xi}(t)) \in \tilde{Q}\left(t_{0}, x_{0}\right) \quad \text { and }|\tilde{\xi}(t)-\xi(t)| \leqslant C\left[\left|x(t)-x_{0}\right|+\left|t-t_{0}\right|\right] \\
\bar{\eta}(t) \leqslant \eta(t)+C\left[\left|x(t)-x_{0}\right|+\left|t-t_{0}\right|\right] \quad \text { for } t \in E, \quad\left|t-t_{0}\right| \leqslant \delta .
\end{gathered}
$$

We say that the sets $\widetilde{Q}(t, x),(t, x) \in A$, have property $\left(\widetilde{F}_{3}^{\prime}\right)$ at the point $\left(t_{0}, x_{0}\right) \in A$, provided

$\left(\widetilde{F}_{3}^{\prime}\right)$ given any number $\sigma>0$, there are constants $C=C\left(t_{0}, x_{0}, \sigma\right)>0$ and $\delta=\delta\left(t_{0}, x_{0}, \sigma\right)>0$ such that for any set of measurable vector functions $x(t), \eta(t)$, $\xi(t), t \in E$, on a measurable set $E \subset G$ with $(t, x(t)) \in A,(\eta(t), \xi(t)) \in \widetilde{Q}(t, x(t))$, $\left|x(t)-x_{0}\right|>\sigma, t \in E,\left|t-t_{0}\right| \leqslant \delta$, there are measurable vector functions $\bar{x}(t), \bar{\eta}(t)$, $\bar{\xi}(t), t \in E$, such that

and

$$
\left(t_{0}, \bar{x}(t)\right) \in A, \quad\left|\vec{x}(t)-x_{0}\right| \leqslant \sigma, \quad(\bar{\eta}(t), \bar{\xi}(t)) \in \widetilde{Q}\left(t_{0}, \bar{x}(t)\right)
$$

$$
\begin{gathered}
|\xi(t)-\bar{\xi}(t)| \leqslant C\left[\left|x(t)-x_{0}\right|+\left|t-t_{0}\right|\right], \\
\vec{\eta}(t) \leqslant \eta(t)+C\left[\left|x(t)-x_{0}\right|+\left|t-t_{0}\right|\right] \quad \text { for } t \in E,\left|t-t_{0}\right| \leqslant \delta .
\end{gathered}
$$


(c) Statements of the Theorems.

THEOREM 1' (A closure theorem). - Let us assume that (i) A is closed, (ii) the sets $\widetilde{Q}(t, x),(t, x) \in A$, are closed and convex, (iii) the sets $\tilde{Q}(t, x),(t, x) \in A$, have property $(Q)$ with respect to $(t, x)$, and property $\left(\tilde{F}_{1}^{\prime}\right)$ with respect to $x$, at every point $\left(t_{0}, x_{0}\right) \in A$, with the exception perhaps of a set of points whose t-coordinates lie in a set $H$ of measure zero in $G$, (iv) if $(\eta, \xi) \in \widetilde{Q}(t, x)$ and $\eta^{\prime}>\eta$ then $\left(\eta^{\prime}, \xi\right) \in \tilde{Q}(t, x)$. Let $\tilde{x}(t)=\left(x^{0}, x\right)$, $\tilde{x}_{k}(t)=\left(x_{k}^{0}, x_{k}\right), t \in G, k \in N$, be given functions with $x, x_{k}: G \rightarrow \boldsymbol{R}^{n}, x^{0}, x_{k}^{0}: \boldsymbol{R}_{0} \rightarrow \boldsymbol{R}$, $R_{0}=\left[a_{0}, b_{0}\right] \supset G$ and assume that $(\mathrm{v}) x_{k}$ are $\mathrm{ACg}, k \in N, x$ is $\mathrm{BVC}$ and $x_{k} \rightarrow \infty$ in $\left(L_{1}(G)\right)^{n}$, (vi) $x_{k}^{0}$ are $\mathrm{VAO}, x^{0}$ is $\mathrm{VBV}$ and $x_{k}^{0} \rightarrow x^{0}$ pointwise a.e. in $R_{0}$. If (vii) $\left(t, x_{k}(t)\right) \in A$ and $\left(D * x_{k}^{0}(t), D x_{k}(t)\right) \in \tilde{Q}\left(t, x_{k}(t)\right)$ a.e. in $G, k \in N$, then

$$
(t, x(t)) \in A \quad \text { and }\left(D^{*} x^{0}(t), D x(t)\right) \in \widetilde{Q}(t, x(t)) \quad \text { a.e. in } G \text {. }
$$

THEOREM $2^{\prime}$ (A closure theorem). - The same as in Theorem 1', where (iii) is replaced by (iii)' the sets $\tilde{Q}(t, x),(t, x) \in A$, have property $\left(\tilde{F}_{2}^{\prime}\right)$ at every point $\left(t_{0}, x_{0}\right) \in A$, with the exception perhaps of a set of points whose t-coordinates lie in a set $H$ of measure zero in $G$.

THEOREM 3' (A closure theorem). - The same as in Theorem 1', where (iii) is replaced by (iii)", the sets $\tilde{Q}(t, x),(t, x) \in A$, have property $(Q)$ with respect to $x$ only and property $\left(\widetilde{F}_{3}^{\prime}\right)$ at every point $\left(t_{0}, x_{0}\right) \in A$, with the exception perhaps of a set of points whose t-coordinates lie on a set $H$ of measure zero in $G$.

\section{(d) Proof of the theorems.}

Proof of Theorem $1^{\prime}$. - We proceed exactly as in the proof of Theorem 1 concerning the functions $x_{k}, x$ with the variants we indicate below. Let us consider the functions $x_{k}^{0}, x^{0}$ which are VAC and VBV respectively, and note that a.e. in $G$ we have

$$
\lim _{h \rightarrow 0^{+}}(2 h)^{-\nu} \Delta_{q} x_{k}^{0}=D^{*} x_{k}^{0}\left(t_{0}\right), \quad h \in N, \quad \lim _{h \rightarrow 0^{+}}(2 h)^{-\nu} \Delta_{q} x^{0}=D^{*} x^{0}\left(t_{0}\right)
$$

where $q=\left[t_{0}-h, t_{0}+h\right]$ with $D^{*} x^{0} \in L_{1}(G), D^{*} x_{k}^{0} \in L_{1}(G), k \in N$.

Thus, for a.a. $t_{0} \in G$ we have

$$
\lim _{h \rightarrow 0^{+}}(2 h)^{-\nu} \int_{q} D^{*} x^{0}(t) d t=D^{*} x^{0}\left(t_{0}\right)
$$

and thus from (1)

$$
\lim _{h \rightarrow 0^{+}}(2 h)^{-\nu}\left(\int_{\boldsymbol{\alpha}} D^{*} x^{0}(i) d t-\Delta_{\boldsymbol{q}} x^{0}\right)=0 .
$$


Moreover, since $x_{k}^{0}$ in $\mathrm{VAC}$, then

$$
\Delta_{\alpha} x_{k}^{0}=\int_{q} D^{*} x_{l k}^{0}(t) d t, k \in \mathbf{N} .
$$

Let us fix now $t_{0}$ as in Theorem 1 and in such a way that (1) and (3) hold and $x_{k}^{0}\left(t_{0}\right) \rightarrow x^{0}\left(t_{0}\right)$ (see hypothesis (vi)). Then we can take $h>0$ as in the proof of Theorem 1 and in such a way that in addition we have

$$
\begin{gathered}
\Delta_{Q} x_{k}^{0} \rightarrow \Delta_{\alpha} x^{0} \quad \text { as } k \rightarrow \infty, \\
\left|(2 h)^{-v} \int_{q} D^{*} x^{0}(t) d t-D^{*} x^{0}\left(t_{0}\right)\right|<\varrho / 7, \\
(2 h)^{-v}\left|\int_{q} D^{*} x^{0}(t) d t-\Delta_{q} x^{0}\right|<\varrho / 7 .
\end{gathered}
$$

Conceming $(5)$, let $t_{0}=\left(t_{0}^{1}, \ldots, t_{0}^{\nu}\right)$, and let $H$ denote the set of all points of $G$ where $x_{k}^{0}$ does not converge to $x^{0}$ as $k \rightarrow \infty$. For every $j=1, \ldots, \nu$, let $\boldsymbol{H}^{j} \subset \boldsymbol{R}^{+}$denote the set of all $l>0$ such that the hyperplanes $t^{j}=t_{0}^{j} \pm l$ intersect $H$ in a set of $(\nu-1)$ dimensional measure zero. Thus, $\boldsymbol{R}^{+}-H^{i}$ has measure zero on $\boldsymbol{R}^{+}$, and we take $\tilde{H}=\bigcap_{j=1}^{v} H^{j}$. For every $h \in \widetilde{H}$ relation (5) holds.

Now we consider an integer $\overline{\bar{k}}=\overline{\bar{k}}\left(t_{0}, x_{0}, \sigma, \varrho, h\right)$ sufficiently large, such that $\overline{\bar{k}}>\bar{k}$, where $\bar{k}$ is fixed as in the proof of Theorem 1 , and moreover for every $k>\overline{\bar{k}}$ we have

$$
(2 h)^{-v}\left|\Delta_{q} x_{k}^{0}-\Delta_{q} x^{0}\right|<\varrho / 7
$$

Finally, from (4), (8) and (7) we obtain

$$
\begin{aligned}
& \left|(2 h)^{-v} \int_{\alpha} D^{*} x_{h}^{0}(t) d t-(2 h)^{-v} \int_{q} D^{*} x^{0}(t) d t\right| \leqslant \\
& \quad \leqslant\left|(2 h)^{-\nu}\left(\Delta_{q} x_{\bar{k}}^{0}-\Delta_{q} x^{0}\right)\right|+\left|(2 h)^{-v}\left(\Delta_{q} x^{0}-\int_{q} D^{*} x^{0}(t) d t\right)\right| \leqslant \varrho / 7+\varrho / 7=2 \varrho / 7 .
\end{aligned}
$$

From (9) and (6), for $k>\overline{\bar{k}}$, we have now

$$
\begin{aligned}
& \left|(2 h)^{-v} \int_{a} D^{*} x_{k}^{0}(t) d t-D^{*} x^{0}\left(t_{0}\right)\right| \leqslant \\
& \leqslant\left|(2 h)^{-v} \int_{a} D^{*} x_{k}^{0}(t) d t-(2 h)^{-v} \int_{a} D^{*} x^{0}(t) d t\right|+\left|(2 h)^{-v} \int_{a} D^{*} x^{0}(i) d t-D^{*} x^{0}\left(t_{0}\right)\right| \leqslant \\
& \leqslant 2 \varrho / 7+\varrho / 7=3 \varrho / 7 .
\end{aligned}
$$

We recall that, for $t \in q \cap K$ and $k>\bar{k}$, then $\left|t-t_{0}\right|<\sigma,\left|x(t)-x\left(t_{0}\right)\right|<\sigma / 2$ and $\left|x_{k}(t)-x(t)\right|<\sigma / 2$. 
Now we proceed as in the proof of Theorem 1 , by enlarging the set $q \cap K$ into the maximal subset $\tilde{K}_{k}$ of $q$ where $\left|x_{k}(t)-x_{0}\right| \leqslant \sigma$, with $x_{0}=x\left(t_{0}\right)$. Note that, for $\eta_{k}(t)=D^{*} x_{k}^{0}(t), \xi_{k}(t)=D x_{k}(t)$, we certainly have (see hypothesis (vii))

$$
\left(\eta_{k}(t), \xi_{k}(t)\right) \in \tilde{Q}\left(t, x_{k}(t)\right), \quad t \in q \text { a.e. . }
$$

By virtue of the property $\left(\tilde{F}_{1}^{\prime}\right)$ corresponding to $\left(t_{0}, x_{0}\right), \sigma>0$ and the functions $x_{k}(t), \eta_{k}(t), \xi_{k}(t), t \in q-\tilde{K}_{k}$, there are measurable vector functions $\bar{x}_{k}(t), \bar{\eta}_{k}(t), \bar{\xi}_{l k}(t)$, $t \in q-\tilde{K}_{k}$, such that

$$
\begin{aligned}
& \left(t, \bar{x}_{k}(t)\right) \in A, \quad\left|\bar{x}_{k}(t)-x_{0}\right| \leqslant \sigma, \quad\left(\bar{\eta}_{k}(t), \bar{\xi}_{k}(t)\right) \in \widetilde{Q}\left(t, \bar{x}_{k}(t)\right), \\
& \left|\xi_{k}(t)-\bar{\xi}_{k}(t)\right| \leqslant C\left[\left|x_{k}(t)-\bar{x}_{k}(t)\right|+\left|t-t_{0}\right|\right], \\
& \bar{\eta}_{k}(t) \leqslant \eta_{k}(t)+C\left[\left|x_{k}(t)-\bar{x}_{k}(t)\right|+\left|t-t_{0}\right|\right], \quad t \in q-\widetilde{K}_{k} .
\end{aligned}
$$

Moreover we can extend $\bar{x}_{k}, \bar{\eta}_{k}$ and $\bar{\xi}_{k}$ to all of $q$, by putting

$$
\bar{x}_{k}(t)=x_{k}(t), \quad \bar{\eta}(t)=\eta_{k}(t), \quad \bar{\xi}_{k}(t)=\xi_{k}(t), \quad t \in \widetilde{K}_{k} .
$$

In the proof of Theorem 1 we already obtained that (see (23) of Section $2 d$ ), for $k>\bar{k}$

$$
\left|D x\left(t_{0}\right)-(2 h)^{-v} \int_{Q} \widetilde{\xi}_{k}(t) d t\right| \leqslant \varrho
$$

with $\left|t-t_{0}\right|<\sigma, \quad\left|\vec{x}_{k}(t)-x_{0}\right| \leqslant \sigma, t \in q$. On the other hand, by (13) and (12) we have

$$
\begin{gathered}
D^{*} x^{0}\left(t_{0}\right)-(2 h)^{-v} \int_{\alpha} \bar{\eta}_{k}(t) d t=D^{*} x^{0}\left(t_{0}\right)-(2 h)^{-\nu} \int_{\tilde{\tilde{K}}_{k}} \eta_{k}(t) d t-(2 h)^{-v} \int_{\alpha-\overline{\bar{K}}_{k}} \bar{\eta}_{k}(t) d t \geqslant \\
\geqslant D^{*} x^{0}\left(t_{0}\right)-(2 h)^{-\nu} \int_{\tilde{\tilde{K}}_{k}} \eta_{k}(t)-(2 h)^{-\nu} \int_{\alpha-\tilde{K}_{k}} \eta_{k}(t) d t-(2 h)^{-\nu} O \int \underset{\alpha-\tilde{K}_{k}}{[}\left[\left|x_{k}(t)-\bar{x}_{k}(t)\right|+\left|t-t_{0}\right|\right] d t= \\
=D^{*} x^{0}\left(t_{0}\right)-(2 h)^{-\nu} \int_{\alpha} \eta_{k}(t) d t-(2 h)^{-\nu} O \int_{\alpha-\tilde{K}_{k}}\left[\left|x_{k}(t)-\bar{x}_{k}(t)\right|+\left|t-t_{0}\right|\right] d t .
\end{gathered}
$$

Therefore by (15), (10) and the third estimate in (22) of Theorem 1 in Section $2 d$, we have, for $k>\overline{\bar{k}}$,

$$
D^{*} x^{0}\left(t_{0}\right)-(2 h)^{-\nu} \int_{Q} \bar{\eta}_{k}(t) d t \geqslant-4 \varrho / 7-3 \varrho / 7=-\varrho .
$$

From (12) and (13) we obtain

$$
\left(\bar{\eta}_{k}(t), \bar{\xi}_{k}(t)\right)_{a} \in \tilde{Q}\left(t, \bar{x}_{k}(t)\right), \quad\left|t-t_{0}\right|<\sigma, \quad\left|\bar{x}_{k}(t)-x_{0}\right| \leqslant \sigma, \quad t \in q,
$$


and therefore

$$
\begin{aligned}
\left((2 h)^{-\nu} \int_{a} \bar{\eta}_{k}(t) d t,(2 h)^{-\nu} \int_{a} \bar{\xi}_{k}(t) d t\right) \in \operatorname{clco} \cup\left[\tilde{Q}\left(t^{\prime}, x^{\prime}\right)|| t^{\prime}-t_{0}|\leqslant \sigma,| x^{\prime}-\right. & \left.x_{0} \mid \leqslant \sigma\right]= \\
& =\operatorname{elco} \widetilde{Q}\left(t_{0}, x_{0}, \sigma\right) .
\end{aligned}
$$

Thus from (14) and (16) we have

$$
\left(D^{*} x^{0}\left(t_{0}\right), D x\left(t_{0}\right)\right) \in\left\{\left(\eta^{\prime}, \xi\right) \mid \eta^{\prime} \geqslant \eta,(\eta, \xi) \in\left[\mathrm{cl} \operatorname{co} \widetilde{Q}\left(t_{0}, x_{0}, \sigma\right)\right]_{e}\right\},
$$

and by the arbitrariety of $\varrho>0$, also

$$
\left(D^{*} x^{0}\left(t_{0}\right), D x\left(t_{0}\right)\right) \in\left\{\left(\eta^{\prime}, \xi\right) \mid \eta^{\prime} \geqslant \eta,(\eta, \xi) \in \mathrm{cl} \operatorname{co} \widetilde{Q}\left(t_{0}, x_{0}, \sigma\right)\right\}
$$

Since $\sigma>0$ is also arbitrary, by property $(Q)$ we obtain (see (iv))

$$
\begin{array}{r}
\left(D^{*} x^{0}\left(t_{0}\right), D x\left(t_{0}\right)\right) \in\left\{\left(\eta^{\prime}, \xi\right) \mid \eta^{\prime} \geqslant \eta,(\eta, \xi) \in \underset{\sigma>0}{ } \operatorname{cl} \operatorname{co} \widetilde{Q}\left(t_{0}, x_{0}, \sigma\right)\right\}= \\
=\left\{\left(\eta^{\prime}, \xi\right) \mid \eta^{\prime} \geqslant \eta,(\eta, \xi) \in \widetilde{Q}\left(t_{0}, x_{0}\right)\right\} \subset \widetilde{Q}\left(t_{0}, x_{0}\right) .
\end{array}
$$

This relation holds a.e. in $K$ with $|G-K|_{p}<\lambda$. Since $\lambda>0$ is arbitrary, we have proved that

$$
\left(D^{*} x^{0}(t), D x(t)\right) \in \tilde{Q}(t, x(t)) \quad \text { a.e. in } G
$$

ProOfs OF THEOREMS $2^{\prime}$ AND $3^{\prime}$. - They are analogous to that of Theorem $1^{\prime}$. See also the proofs of Theorems 2 and 3 in Section $2 d$.

\section{4. - The existence theorems.}

(a) The integral J.

Let $y \geqslant 1, n \geqslant 1,0 \leqslant \alpha \leqslant n, N \geqslant 1$ be integers and, for every $x \in \boldsymbol{R}^{n}$, let us write $x=(y, z)$ with $y \in \boldsymbol{R}^{\alpha}$ and $z \in \boldsymbol{R}^{n-\alpha}$. Let $A, G$ and $Q(t, x)$ be given sets as in Section 2.

We recall that, for every $\mathrm{BVC}$ vector function $x(t)=\left(x^{1}, \ldots, x^{n}\right), t \in G$, we denote by $D x(t)$ the $N$-vector function $D^{j} x^{i}(t), j \in\{j\}_{i}, i=1, \ldots, n$. We shall also consider two other systems of indices $j\left[\{j\}_{i}^{(1)}, i=1, \ldots, \alpha\right]$ and $\left[\{j\}_{i}^{(2)}, i=\alpha+1, \ldots, n\right]$, $1 \leqslant j_{1}<\ldots<j_{s} \leqslant \nu$, and corresponding systems of derivatives

$$
\begin{aligned}
& D^{(1)} y(t)=\left\{D^{i} x^{i}(t), j \in\{j\}_{i}^{(1)}, i=1, \ldots, \alpha\right\}, \\
& D^{(2)} z(t)=\left\{D^{i} x^{i}(t), j \in\{j\}_{i}^{(2)}, i=\alpha+1, \ldots, n\right\} .
\end{aligned}
$$

Let $N_{1}$ and $N_{2}$ be the total number of indices contained in $\{j\}_{i}^{(1)}, i=1, \ldots, \alpha$, and $\{j\}_{i}^{(2)}, i=\alpha+1, \ldots, n$, respectively. Then $N=N_{1}+N_{2}$. Let $\boldsymbol{M} \subset \boldsymbol{R}^{\nu+n+N}$ denote 
the set $M=\{(t, x, \xi) \mid(t, x) \in A, \xi \in Q(t, x)\}$ and let $F_{0}(t, x, \xi)$, or $F_{0}: M \rightarrow \boldsymbol{R}$, denote a given real valued function on $M$. Let $\Omega$ be a class of admissible vector functions $x(t)=(y(t), z(t))$, or $x: G \rightarrow \boldsymbol{R}^{n}$, that is, satisfying

(i) $y$ is $\mathrm{ACg}$ and $z$ is $\mathrm{BVO}$ on $G$,

(ii) $(t, x(t)) \in A, D x(t) \in Q(t, x(t))$,

(iii) $F_{0}(\cdot, x(\cdot), D x(\cdot)) \in L_{1}(G)$.

For any element $x=(y, z) \in \Omega$, let $\Gamma(x)$ be the class of all sequences $\left(x_{k}\right)_{k \in N}$, with $x_{k}: G \rightarrow \boldsymbol{R}^{n}$ such that

(a) $x_{k}=\left(y_{k}, z_{k}\right) \in \operatorname{ACg} \cap \Omega, k \in \mathbb{N}$,

(b) $x_{k} \rightarrow x$ in $\left(L_{1}(G)\right)^{n}, D^{j} y_{b}^{i} \rightarrow D^{j} y^{i}$ weakly in $L_{1}(G), j \in\{j\}_{i}^{(1)}, i=1, \ldots, \alpha$.

We consider the functional $\mathfrak{J}: \Omega \rightarrow \widetilde{R}$ defined by

$$
\begin{aligned}
& J(x)=J(y, z)=\inf _{\Gamma(x)} \lim _{\hat{l} \rightarrow \infty} \int_{G} F_{0}\left(t, x_{k}(t), D x_{k}(t)\right) d t=\inf _{\Gamma(x)} \lim _{h \rightarrow \infty} I\left(x_{k}\right) \quad \text { if } \Gamma(x) \neq \emptyset \\
& \text { and } J(x)=+\infty \text { if } \Gamma(x)=\emptyset .
\end{aligned}
$$

The functional $\mathfrak{J}$ is modeled on Lebesgue area theory for nonparametric discontinuous surfaces (see CESARI [5]) and it is also close to the concept of integral in the sense of Serrin ([24]) when the present mixed convergence is used.

The class $\Omega$ is said to be closed if $\Omega$ has the following property: for any sequence $\left(x_{k}\right)_{k \in N}$ of pairs in $\Omega$ such that $(a)$ and $(b)$ are satisfied and the limit $x=(y, z)$ satisfies (i) (ii), (iii), then $x \in \Omega$.

REMARK 1. - In order to deal with the functional under consideration in the case that either traces or boundary data are given, we need to carry over the definition of the functional $J$ in a slightly different setting. For instance, if the trace $\varphi$ is given on $\partial G$, with $G$ possessing the cone property (see Section $4 c$ below) and $J^{r-1}(\partial G)<$ $<+\infty$, we may restrict the class $\Gamma(x)$ to those sequences $\left(x_{k}\right)$ which are in $\mathrm{ACg} \cap \Omega$ and such that $x_{k} \rightarrow x$ in $\left(L_{1}(G)^{n}\right), D^{j} y_{k}^{i} \rightarrow D^{j} y^{i}$ weakly in $L_{1}(G), \gamma\left(x_{k}\right)=\varphi^{\prime}$ and $V^{*}\left(x_{k}\right) \rightarrow V^{*}(x)$ as stated in [11a].

Then the new Serrin-type integral, say $J^{*}(x)$, satisfies $J^{*}(x) \geqslant J(x)$, and by a theorem due to Anzellotti-Giaquinta ([2]), $\gamma(x)=\varphi$.

Instead, if boundary data on $\partial G$ are given, then under the same assumptions on $G$, we may take for $\Omega$ the class of functions $x: \bar{G} \rightarrow \boldsymbol{R}^{n}$, which are BVC when disregarding sets $E \subset G,|E|_{\nu}=0$, and where the variations $V^{*}(x, \bar{G})$ (see section 2.a) are computed taking note of the values of $x$ on the boundary (cfr. [11b], Remark 2). 
(b) A lower semicontinuity property of $I$ and $J$.

The lowersemicontinuity of the Serrin integral $J$ is well known.

As an application of the closure theorems of Section 3 we shall prove now the semicontinuity of the integral functional $I$. In order to do that, let us consider the "augmented"sets

$$
\tilde{Q}(t, x)=\left\{\left(z^{0}, \xi\right) \in \boldsymbol{R}^{N+1}: z^{0} \geqslant F_{0}(t, x, \xi), \xi \in Q(t, x)\right\}, \quad(t, x) \in A
$$

Theorem $1^{\prime \prime}$ (A lower semicontinuity theorem). - Let $0 \leqslant \alpha \leqslant n$, and assume that: (i) $A$ is closed, (ii) the sets $\tilde{Q}(t, x),(t, x) \in A$, are closed, convex and satisfy property $(Q)$ with respect to $(t, x)$, and property $\left(\widetilde{F}_{1}^{\prime}\right)$ with respect to $x$, at every point $\left(t_{0}, x_{0}\right) \in A$, with the exception perhaps of a set of points whose $t$-coordinates lie on a set $H$ of measure zero in the $t$-space, (iii) $F_{0}(t, x, \xi)$ is lower semicontinuous on $M$ and $F_{0}(t, x, \xi) \geqslant \lambda(t)$ with $\lambda \in L_{1}(G)$ for all $(t, x, \xi) \in M$.

We assume also that (iv) a sequence of vector functions is given $x(t)=(y, z)$, $x_{k}(t)=\left(y_{k}, z_{k}\right), t \in G$, such that $y, y_{k}, z_{k}$ are $\mathrm{ACg}, z$ is $\mathrm{BVC}, x_{k} \rightarrow x$ in $\left(L_{1}(G)\right)^{n}$ and $\left(t, x_{k}(t)\right) \in A, D x_{k}(t) \in Q\left(t, x_{k}(t)\right)$ a.e. in $G, k \in N$. Then $(t, x(t)) \in A, D x(t) \in Q(t, x(t))$ a.e. in $G$, and

$$
\lim _{k \rightarrow \infty} I\left(x_{k}\right) \geqslant I(x)
$$

Thus, if $\left(x_{k}\right)_{k \in N}$ is a sequence as in the definition of $J(x)$, then

$$
\lim _{k \rightarrow \infty} I\left(x_{k}\right) \geqslant J(x) \geqslant I(x)
$$

THeOREM 2" (A lower semicontinuity theorem). - The same as in Theorem $1^{\prime \prime}$, where (ii) is replaced by (ii) ${ }^{t}$ the sets $\tilde{Q}(t, x),(t, x) \in A$, are closed and convex and satisfy property $\left(\tilde{E}_{2}^{\prime}\right)$ at every point $\left(t_{0}, x_{0}\right) \in A$, with the exception perhaps of a set of points whose $t$-coordinates lie in a set $H$ of measure zero in $G$.

Theorem $3^{\prime \prime}$ (A lower semicontinuity theorem). - The same as in Theorem $1^{\prime \prime}$, where (ii) is replaced by (ii) the sets $\tilde{Q}(t, x),(i, x) \in A$, are closed and convex and satisfy property $(Q)$ with respect to $x$ only, and property $\left(\tilde{F}_{3}^{\prime}\right)$ at every point $\left(t_{0}, x_{0}\right) \in A$, with the exception perhaps of a set of points whose t-coordinates lie on a set $H$ of measure zero in $G$.

Proof of THEOREM $1^{\prime \prime}$. W.l.o.g. we can suppose that

$$
i=\lim _{k \rightarrow \infty} I\left(x_{k}\right)=\lim _{k \rightarrow \infty} I\left(x_{k}\right)<+\infty
$$

where $i$ is finite because of (iii). Let $R_{0}=\left[a_{0}, b_{0}\right], a_{0}=\left(a_{0}^{1}, \ldots, a_{0}^{v}\right), b_{0}=\left(b_{0}^{1}, \ldots, b_{0}^{v}\right)$, $a_{0}^{i}<b_{0}^{i}$ be any closed interval containing $\mathrm{cl} G$ in its interior. Iset us consider the 
functions $F_{k}(t)=F_{\mathbf{0}}\left(t, y_{k}(t), z_{k}(t), D^{(1)} y_{k}(t), D^{(2)} z_{k}(t)\right), t \in G \subset \boldsymbol{R}^{v}$, and let us extend $F_{k}$ to all of $R_{0}$ by taking

$$
\varphi_{k}(t)= \begin{cases}F_{k}(t), & t \in G \\ 0, & t \in R_{0}-G\end{cases}
$$

Now let $x_{k}^{0}: R_{0} \rightarrow \boldsymbol{R}$ be defined by

$$
x_{k}^{0}(t)=\int_{\left[a_{0}, t\right]} \varphi_{k}(\tau) d \tau, \quad k \in N, \quad d \tau=d \tau^{1} \ldots d \tau^{v}
$$

Each function $x_{k}^{0}$ is VAC, $k \in N$. Moreover, $x_{k}^{0}\left(a_{0}\right)=0$,

$$
V_{j}\left(x_{k}^{\mathbf{0}}\left(\cdot, a_{0}^{\prime j}\right) ;\left[a_{0}^{j}, b_{\mathbf{0}}^{j}\right]\right)=0, \quad j=1, \ldots, v, \quad \text { and } \sup _{D} \sum_{R \in D}\left|A_{n} x_{k}^{0}\right| \leqslant W, \quad k \in \mathbf{N} .
$$

In order to prove this last assertion, observe that for $k$ sufficiently large

$$
\begin{aligned}
\sum_{R \in D}\left|\Delta_{R} x_{k}^{0}\right|= & \sum_{R \in D}\left|\int_{R} \varphi_{k}(t) d t\right|<\sum_{R \in D}\left[\int_{R}\left(\varphi_{k}(t)-\lambda(t)\right) d t+\int_{R}|\lambda(t)| d t\right] \leqslant \\
& \leqslant \int_{R_{0}} \varphi_{k}(t) d t+2 \int_{R_{0}}|\lambda(t)| d t=I\left(x_{k}\right)+2 \int_{G}|\lambda(t)| d t \leqslant i+1+2 \int_{G}|\lambda(t)| d t=W,
\end{aligned}
$$

where we extended $\lambda$ to all of $R_{0}$ by taking $\lambda(t)=0$ on $R-G$. Therefore, by Helly's theorem for functions of bounded variations in the sense of Vitali, (see [18], pg. 115), there exists a subsequence, say still [k], such that $x_{k}^{0} \rightarrow x^{0}$ pointwise on $R_{0}$, with $x^{0}$ VBV. Note that the sequence $\left(x_{k}^{0}, x_{k}\right)$ satisfies the orientor field equation

$$
\left(t, x_{k}(t)\right) \in A, \quad\left(D^{*} x_{k}^{0}(t), D x_{k}(t)\right) \in \widetilde{Q}\left(t, x_{k}(t)\right), \quad t \in G \text { a.e.. }
$$

Thus, by the closure Theorem $1^{\prime}$ of Section $3 c$, we have

$$
(t, x(t)) \in A, \quad\left(D^{*} x^{0}(t), D x(t)\right) \in \widetilde{Q}(t, x(t)) \quad \text { a.e. in } G .
$$

Hence,

$$
D^{*} x^{0}(t) \geqslant F_{0}(t, x(t), D x(t)) \geqslant \lambda(t), \quad t \in G,
$$

and therefore $F_{0}(\cdot, x(\cdot), D x(\cdot)) \in L_{1}(G)$.

Finally, if we take $X^{0}(t)=x^{0}(t)-\int_{[a, t]} \lambda(\tau) d \tau$, then $X^{0} \geqslant 0, X^{0}$ is VBV and $D^{*} X^{0}(t)=$ $=D^{*} x^{0}(t)-\lambda(t) \geqslant 0$ a.e.in $R_{0}$. Moreover (see RADo [21] III.1.28),

$$
\Delta_{R_{0}} X^{0}=x^{0}(b)-\int_{R_{0}} \lambda(t) d t \geqslant \int_{R_{0}} D^{*} X^{0}(t) d t \geqslant \int_{G} D^{*} x^{0}(t) d t-\int_{G} \lambda(t) d t .
$$


Thus

$$
I(x)=\int_{G} F^{\prime}(t, x(t), D x(t)) d t \leqslant \int_{G} D^{*} x^{0}(t) d t \leqslant x^{0}(b)=\lim _{k \rightarrow \infty} x_{h}^{0}(b)=\lim _{k \rightarrow \infty} I\left(x_{h}\right) .
$$

PRoOfs of THeOREms 2" AND $3^{\prime \prime}$. - They are the same as those of Theorem $1^{\prime \prime}$, bu virtue of the Theorems $2^{\prime}$ and $3^{\prime}$ respectively.

REILARK 2. - As an immediate consequence of Theorem $1^{\prime \prime}$ (or $2^{\prime \prime}$ and $3^{\prime \prime}$ ) we see that, under the assumption considered there, the functional $J$ is an extension to BVC of the functional $I$ on $\mathrm{ACg}$. In fact, $I(x) \leqslant J(x)$ for every $x \in \Omega$, moreover if $x \in \mathrm{ACg} \cap \Omega$, then $\Gamma(x)$ contains the sequence of repetitions $x_{k}=x, k \in \mathbb{N}$, hence $J(x) \leqslant I(x)$ and finally $I(x)=J(x)$.

\section{(c) Statements of the existence Theorems.}

In the following we shall use the growth conditions below.

$\left(\gamma_{1}\right)$ There is a sealar function $\phi(\zeta), 0 \leqslant \zeta<+\infty$, or $\phi: \mathbb{R}_{0}^{+} \rightarrow \boldsymbol{R}$, bounded below, with $\phi(\zeta) / \zeta \rightarrow+\infty$ as $\zeta \rightarrow+\infty$, such that $F_{0}(t, x, u, v) \geqslant \phi(|u|)$ for all $(t, x, u, v) \in M$.

$\left(\gamma_{2}\right)$ For any $\varepsilon>0$ there is an integrable scalar function $\psi_{\varepsilon}(t)>0$, or $\psi_{\varepsilon}: G \rightarrow \boldsymbol{R}_{0}^{+}$, such that $|u| \leqslant \psi_{\varepsilon}(t)+\varepsilon F_{0}(t, x, u, v)$ for all $(t, x, u, v) \in M$.

$\left(\gamma_{3}\right)$ for any $\alpha$-vector $p \in \boldsymbol{R}^{\alpha}$ there is an integrable scalar function $\phi_{p}(t)>0$, or $\phi_{p}: G \rightarrow \boldsymbol{R}_{0}^{+}$, such that $F_{0}(t, x, u, v) \geqslant\langle p, u\rangle-\phi_{p}(t)$ for all $(t, x, u, v) \in M$.

Note that, under condition $\left(\gamma_{1}\right)$ certainly $\phi(\zeta) \geqslant \lambda$ for some real constant $\lambda$, and then $F_{0}(t, x, u, v) \geqslant \phi(|u|) \geqslant \lambda$ for all $(t, x, u, v)$. Under condition $\left(\gamma_{2}\right)$ and $\varepsilon=1$ we have $|u| \leqslant \psi_{1}(t)+F_{0}(t, x, u, v)$, hence $F_{0}(t, x, u, v) \geqslant-\psi_{1}(t)$, a function in $L_{1}(G)$. Under condition $\left(\gamma_{3}\right)$ and $p=0$, we have $F_{0}(t, x, u, v)>-\phi_{0}(t)$, a function in $L_{1}(G)$.

BEMARI 3. - Note that for every $x \in A$ Cg $\cap \Omega$ then $\Gamma(x) \neq \emptyset$ and $J(x)<+\infty$. Moreover, if we suppose $\operatorname{ACg} \cap \Omega \neq \emptyset$, then

$$
-\infty \leqslant \inf _{\Omega} J(x)<+\infty \quad \text { and } i=\inf _{\Omega} J(x)=\inf _{A \operatorname{con} \Omega} I(x)
$$

In order to see this, note first that if $i=\inf _{\Omega} J(x)$, we also have

$$
i \inf _{A C g \cap \Omega} J(x)=\inf _{A C g \cap \Omega} I(x)
$$

Then, it is easy to see that, for every $x \in \Omega$, there is a sequence $\left(x_{m}\right)_{m \in N}$ in $\operatorname{ACg} \cap \Omega$ such that $J(x)=\lim _{m \rightarrow \infty} I\left(x_{m}\right)$. As a consequence, we can find a sequence $\left(x_{k}\right)_{k \in N}$ in 
$\operatorname{ACg} \cap \Omega$ such that $i=\lim _{k \rightarrow \infty} I\left(x_{k}\right)$. Thus

$$
\inf _{A C g \cap \Omega} I(x) \leqslant i .
$$

We recall here some well-known conditions on the set $G$ that we shall use in the following.

The set $G \subset \boldsymbol{R}^{r}$ is said to have the cone property if there exists a finite cone $C$ in $\boldsymbol{R}^{v}$ such that each point $t \in \partial G$ is the vertex of a finite cone $C_{t}$ contained in $G$ and congruent to $C$. The bounded set $G \subset \boldsymbol{R}^{y}$ is said to have a locally Lipschitz boundary if each point $t \in \partial G$ has a neighborhood $U_{t}$ such that $\partial G \cap U_{t}$ is the graph of a Lipschitz function.

The following well-known result by GAGLIARDo [16] states a relevant property of the open sets with the cone property.

THeOReMr (Gagliardo [16]). - If $G$ is a bounded open set in $\boldsymbol{R}^{v}$, with the cone property, then there is a finite collection $G_{1}, \ldots, G_{m}$ of open sets with $G=\bigcup_{l=1}^{m} G_{l}$, such that
each $G_{l}$ has a locally Lipschitz boundary.

THEOREM 4. (An existence theorem). - Let $1 \leqslant \alpha \leqslant n-1$ and assume that

(i) $G$ has the cone property, $A$ is compact and $M$ is olosed; (ii) the sets $\widetilde{Q}(t, x)$, $(t, x) \in A$, are closed, convex and satisfy property $(Q)$ with respect to $(t, x)$, and property $\left(\widetilde{F}_{1}^{\prime}\right)$ with respect to $x$, at every point $\left(t_{0}, x_{0}\right) \in A$, with the exception perhaps of $a$ set of points whose t-coordinates lie on a set $H$ of measure zero in the $t$-space; (iii) $F_{0}(t, x, \xi)$ is lower semicontinuous on $M$; (iv) $F_{0}$ satisfies one of the growth conditions $\left(\gamma_{1}\right),\left(\gamma_{2}\right)$ or $\left(\gamma_{3}\right)$. We assume atso that $\Omega$ is closed, $\operatorname{ACg} \cap \Omega \neq \emptyset$ and $(\mathrm{v})$ there are constants $p>1$ and $V_{0}$ such that $\int_{G}\left|D^{j} y^{i}(t)\right|^{p} d t \leqslant V_{0}$, for all $j \notin\{j\}_{i}^{(1)}, i=1, \ldots, \alpha$; (vi) there is a constant $W_{0}$ such that $V^{*}\left(z^{i}\right) \leqslant W_{0}, i=\alpha+1, \ldots, n$.

Then the functional $\mathfrak{J}$ has an absolute minimum $x=(y, z) \in \Omega, y \in \mathrm{ACg}, z \in \mathrm{BVC}$. For $\alpha=0$, then $x=z$, requirements $\left(\gamma_{1}\right)$ or alternates $\left(\gamma_{2}\right),\left(\gamma_{3}\right)$ and condition $(v)$ do not apply, yet the conolusion is still valid if we know that (iv)' there is a summable scalar function $\lambda: G \rightarrow \boldsymbol{R}$ such that $F_{0}(t, z, \xi) \geqslant \lambda(t)$, for all $(t, z, \xi) \in M$.

For $\alpha=n$, then $x=y, \Omega$ is a non empty and closed class of ACg functions $y(t)=$ $=\left(y^{1}, \ldots, y^{n}\right), t \in G$, conditions (vi) does not apply, yet the conclusion is still valid.

Note that the condition (ii) concerning property $(Q)$ for the sets $\widetilde{Q}(t, x)$ not only implies that the same sets $\widetilde{Q}(t, x)$ are closed and convex, but also that their projections, the sets $Q(t, x)$, also are convex, and that $F_{0}(t, y, z, u, v)$ is convex in $(u, v)$ on each $Q(t, y, z)$.

THeorex 5 (An existence theorem). - The same as in Theorem 4 where (ii) is replaced by (ii)' the sets $\widetilde{Q}(t, x),(t, x) \in A$, are closed, convex and satisfy properiy $\left(\widetilde{F}_{2}^{\prime}\right)$ 
at every point $\left(t_{0}, x_{0}\right) \in A$ with the exception perhaps of a set of points whose t-coordinates lie in a set $H$ of measure zero in $G$.

Theorern 6 (An existence theorem). - The same as in Theorem 4 where (ii) is replaced by (ii)" the sets $\widetilde{Q}(t, x),(t, x) \in A$, are closed, convex and satisfy property $(Q)$ with respect to $x$ only, and property $\left(\tilde{F}_{3}^{\prime}\right)$ at every point $\left(t_{0}, x_{0}\right) \in A$ with the exception perhaps of a set of points whose $t$-coordinates lie in a set $H$ of measure zero in $G$.

Proof of Theorwa 4. - We already know (see Remark 2 ) that $i=\inf _{\Omega} J(x)=$ $=\inf _{A C g \cap \Omega_{\mathrm{xy}}} I(x)$, and since $\Omega \cap \mathrm{ACg} \neq \emptyset$, we also have $-\infty \leqslant i<+\infty$.

Under either assumption in (iv) there is a function $\lambda \in L_{1}(\theta)$ such that

$$
I(x)=\int_{G} F_{0}(t, x(t), D x(t)) d t \geqslant \int_{G} \lambda(t) d t>-\infty .
$$

Thus, $-\infty<i<+\infty$, and we consider a minimizing sequence $\left(x_{k}\right) \subset \operatorname{ACg} \cap \Omega$ with $I\left(x_{k}\right) \rightarrow i$. We may well assume $i \leqslant I\left(x_{k}\right) \leqslant i+1, k \in N$. By virtue of assumption $\left(\gamma_{1}\right)$, we derive that

$$
i+1 \geqslant I\left(x_{k}\right)=\int_{G} F_{0}\left(t, y_{k}(t), z_{k}(t), D^{(1)} y_{k}(t), D^{(2)} z_{k}(t)\right) d t \geqslant \int_{G} \phi\left(\left|D^{(1)} y_{k}(t)\right|\right) d t
$$

By Nagumo's theorem (cfr. [8], 10.3.i), we derive that the vector functions $D^{(1)} y_{k}(t)$ are equiabsolutely integrable in $G$. By 10.4.ii and 10.4.iii in [8] we derive the same conclusion under assumptions $\left(\gamma_{2}\right)$ or $\left(\gamma_{3}\right)$ respectively. By assumption (v) we know that the partial derivatives $D^{j} y_{k}^{i}, j \notin\{j\}_{i}^{(1)}, i=1, \ldots, \alpha$, are equiabsolutely integrable in $G$. Thus this holds for all derivatives $D^{i} y^{i}, j=1, \ldots, v, i=1, \ldots, \alpha$. Since the functions $y_{k}^{i}$ are $\mathrm{ACg}$, we conclude that the total variations $V_{0}\left(y_{k}^{i}\right)$, $i=1, \ldots, \alpha, k \in N$, are equibounded.

Note that, since $A$ is bounded, the functions $\left(x_{k}\right)$ are equibounded in $\left(L_{1}(G)\right)^{n}$.

Thus, as a consequence of (vi) and the cone property of $G$, by virtue of the compactness result due to CAFIERo-FLEMING ([4], [15], see also [3] pg. 169-153 and [1] pg. 146), there is a sub-sequence, say still $(k)$, such that

$$
x_{k} \rightarrow x \text { in }\left(L_{1}(G)\right)^{n} \quad \text { with } x=(y, z) \in \mathrm{BVC} .
$$

Moreover, by the equi-absolute-integrability of $D^{j} y^{i}, j=1, \ldots, v, i=1, \ldots, \alpha$, from Theorem 10.4.i of [8] we conclude, again for a suitable subsequence, that

$$
D^{j} y_{k}^{i} \rightarrow D^{j} y^{i} \text { weakly in } L_{1}(G), \quad j=1, \ldots, v, i=1, \ldots, \alpha
$$

and $y$ is ACg. 
Now, if we proceed as in the proof of Theorem $1^{\prime \prime}$, we prove that $x=(y, z) \in \Omega$ and $\left(x_{k}\right) \in \Gamma(x)$. Hence,

$$
i \leqslant J(x) \leqslant \varliminf_{k \rightarrow \infty} J\left(x_{k}\right)=\varliminf_{k \rightarrow \infty} I\left(x_{k}\right)=i
$$

and the proof is complete.

Proofs of Theorems 5 AND 6. - They are analogous to that of Theorem 4.

RELARK 4. - The hypothesis (vi) can be replaced by the lighter assumption (vi) the level sets $L_{k}=\{x=(y, z) \in \operatorname{ACg} \cap \Omega: I(x) \leqslant K\}$ are equibounded in variation. In fact, as it can be seen by the proof, we use condition (vi) only to guarantee that every minimizing sequence of $\mathrm{ACg}$ surfaces $x=(y, z)$ is equibounded in variation.

REMARK 5. - Note that the condition that $A$ is bounded can be dropped if we know that there is a minimizing sequence $x_{k}=\left(y_{k}, z_{k}\right), y_{k}, z_{k} \in \mathrm{AOg}$, with $I\left(x_{k}\right) \rightarrow i$, which is equibounded in $L_{1}$.

We shall give now an Example which illustrates Theorem 4 and shows that, in general, the minimum of $J$ is attained by a BVO function, not necessarily $A C g$.

Exampte 1. - Let us take

$$
G=(0,2)^{2}, \quad A=\left\{\left(t_{1}, t_{2}, z\right) \in[0,2]^{3}: t_{1}-1 / 2 \leqslant z \leqslant t_{1}+1 / 2\right\}, \quad Q\left(t_{1}, t_{2}, z\right)=\boldsymbol{R}^{2}
$$

and

$$
I(z)=\int_{G}\left\{\left(\left(1-t_{1}\right) z_{t_{1}}\right)^{2}+\left(\left(1-t_{2}\right) z_{t_{2}}\right)^{2}\right\}^{1 / 2} d t_{1} d t_{2}
$$

Thus, $F_{0}: G \times \boldsymbol{R}^{2} \rightarrow \boldsymbol{R}$ is defined by

$$
F_{0}\left(t_{1}, t_{2}, v_{1}, v_{2}\right)=\left\{\left[\left(1-t_{1}\right) v_{1}\right]^{2}+\left[\left(1-t_{2}\right) v_{2}\right]^{2}\right\}^{1 / 2}
$$

Let $\Omega$ be the class of all the BVC surfaces $z:(0,2)^{2} \rightarrow \boldsymbol{R}$. Let us prove that $\min _{\Omega} J(z)=0=J(\bar{z})=i$, where

$$
\bar{z}\left(t_{1}, t_{2}\right)=\left\{\begin{array}{cl}
\frac{1}{2}, & t_{1} \in(0,1) \\
1, & t_{1}=1, \\
\frac{3}{2}, & t_{1} \in(1,2)
\end{array}\right.
$$


In fact, the sequence $z_{k}:(0,2)^{2} \rightarrow \boldsymbol{R}$ of AOT surfaces defined by

$$
z_{k}\left(t_{1}, t_{2}\right)=\left\{\begin{array}{l}
\frac{1}{2}, \quad t_{1} \in(0,1-1 / k], \\
(k / 2) t_{1}-(k / 2)+1, \quad t_{1} \in(1-1 / k, 1+1 / k), \\
\frac{3}{2}, \quad t_{1} \in[1+1 / k, 2)
\end{array}\right.
$$

converges in $L_{1}$ to $\bar{z}$, and

$$
I\left(z_{k}\right)=2 \int_{-1 / k}^{1+1 / k} \mid 1-t / k / 2 d i=k\left(1 / k^{2}\right) \rightarrow 0, \quad J(\vec{z})=0 .
$$

The minimum $i=0$ is attained by $J$ also at any sectionally constant function $z$ on $(0,2)$ whose graph is in $A$. Note that this minimum cannot be attained at any $\mathrm{ACg}$ function. In fact, in this ease, we would have $J(\overline{\bar{z}})=I(\overline{\bar{z}})=0$ with $\overline{\bar{z}} \mathrm{ACg}$, hence, $\overline{\bar{z}}_{t_{1}}=0, \overline{\bar{z}}_{t_{2}}=0$ a.e. in $G$, and $\overline{\bar{z}}$ would be constant in $G$, but this is not possible by the shape of $A$.

\section{REFERENCES}

[1] R. A. ADAMS, Sobolev Spaces, Academic Press (1975).

[2] G. Anzellotti - M. Giaquinta, Funzioni BV e tracce, Rend. Sem. Mat. Univ. Padova, 60 (1978), pp. 1-21.

[3] H. BREzIs, Analyse Fonctionnelle - Theorie et Applications, Masson (1984).

[4] F. CAIIERo, Oriteri di compattezza per le successioni di funzioni generalmente a variazione limitata, Rend. Accad. Naz. Lincei, 8 (1950), pp. 305-310.

[0] L. Cesari, Sulle funzioni a variazione limitata, Annali Scuola Norm. Sup. Pisa, 5 (1936), pp. 299-313.

[6] L. CESARI, Existenee theorems for multidimensional problems of optimal control, Differ. ential Equations and Dynamical Systems, Academic Press (1967), pp. 115-132.

[7] L. Cesari, Existence theorems for multidimensional Lagrange problems, J. Optimization Theory Appl., 1. (1967), pp. 87-112.

[8] L. CESARI, Optimization-Theory and Applications, Springer-Verlag (1983).

[9] L. Cesari, Nonlinear analysis, Boll. Un. Mat. Ital,, 6 (1985), pp. 157-216.

[10] L. CESARI, a) Sulle funzioni di due variabili a variazione limitata e sulla convergenza delle relative serie doppie di Fourier, Rend. Sem. Mat. Univ. Roma, (4), 1. (1937), pp. 277-294; b) Sulle funzioni di piu variabili a variazione limitata e sulla convergenza delle relative serie multiple di Fourier, Pont. Accad. Scienze, Commentationes, 3 (1939), pp. 171-197.

[11] L. Cegari - P. BRANDI - A. SALVADORI, a) Discontinuous solutions in problems of opiimization, Annali Scuola Normale Sup. Pisa, to appear; b) Existence theorems concerning simple integrals of the calculus of variations for discontinnous solutions, Archive Rat. Mech. Anal., 98 (1987), pp. 307-328.

[12] E. CONWAY - J. SMoller, Global solutions of the Oauchy problem for quasi linear first order equations in several variables, Comm. Pure Appl. Math., 19 (1966), pp. 95-105. 
[13] C. M. Dafermos, Characteristics in hyperbolic conservation law. A study of all structure and the asymptotic behaviour of solutions, Nonlinear Analysis and Mechanics, Watt Sym. posium, Pitman, I. (1977), pp. 1-58.

[14] R.J. Di PERna, Singularities of solutions of nonlinear hyperbolic systems of conservation laws, Arch. Rat. Mech. Anal., 60 (1975), pp. 75-100.

[15] W. H. Flemng, Functions whose partial derivatives are measures, Illinois J. Math., 4 (1960), pp. 452-478.

[16] E. GaGLIARDo, Proprietà di alcune funzioni in più variabili, Ricerche Mat., 7 (1958), pp. 102-137.

[17] E. GIUSTI, Minimal Surfaces and Functions of Bounded Variation, Monographs in Mathematics, 80, Birkhauser (1984).

[18] T. H. Hildebrandt, Theory of Integration, Academic Pross (1963).

[19] K. KRICKEBERG, Distributionen, Funktionen beschränkter Variation und Lebesguescher Inhalt nichtparametrischer Flächen, Annali Mat. Pura Appl., (IV), 44 (1957), pp. 92, 105-133.

[20] M. MrRanda, Distribuzioni aventi derivate misure. Insiemi di perimetro localmente finito Annali Scuola Norm. Sup. Pisa, (3), 19 (1964), pp. 27-56.

[21] T. Liadò, tength and Area, Amer. Math. Soe. Colloquium Publ., 30 (1950), pp. 8, 305-310.

[22] S. SAKs, Theory of the Integral, Monografie Matematyczne, 7, Hafner Publ. (1937).

[23] J. SERRIN, On the differentiability of funetions of several variables, Arch. Rat. Mech. Anal., 7 (1961), pp. 359-372.

[24] J. SERRIN, On the definition and properties of certain variational integrals, Trans. Amer. Math. Soc., 101 (1961), pp. 139-167. 